\title{
Common Fixed Point Theorems in Modified Intuitionistic Fuzzy Metric Spaces
}

\author{
Saurabh Manro, ${ }^{1}$ Sanjay Kumar, ${ }^{2}$ S. S. Bhatia, ${ }^{1}$ and Kenan Tas $^{3}$ \\ ${ }^{1}$ School of Mathematics and Computer Applications, Thapar University, Patiala, Punjab, India \\ ${ }^{2}$ Deenbandhu Chhotu Ram University of Science and Technology, Murthal, Sonepat, India \\ ${ }^{3}$ Cankaya University, Department of Mathematics and Computer Science, Ankara, Turkey
}

Correspondence should be addressed to Saurabh Manro; sauravmanro@hotmail.com

Received 20 December 2012; Revised 11 February 2013; Accepted 13 February 2013

Academic Editor: Reinaldo Martinez Palhares

Copyright (C) 2013 Saurabh Manro et al. This is an open access article distributed under the Creative Commons Attribution License, which permits unrestricted use, distribution, and reproduction in any medium, provided the original work is properly cited.

\begin{abstract}
This paper consists of main two sections. In the first section, we prove a common fixed point theorem in modified intuitionistic fuzzy metric space by combining the ideas of pointwise $R$-weak commutativity and reciprocal continuity of mappings satisfying contractive conditions. In the second section, we prove common fixed point theorems in modified intuitionistic fuzzy metric space from the class of compatible continuous mappings to noncompatible and discontinuous mappings. Lastly, as an application, we prove fixed point theorems using weakly reciprocally continuous noncompatible self-mappings on modified intuitionistic fuzzy metric space satisfying some implicit relations.
\end{abstract}

\section{Introduction and Preliminaries}

Recently, Saadati et al. [1] introduced the modified intuitionistic fuzzy metric space and proved some fixed point theorems for compatible and weakly compatible maps. Consequently, in this modified setting of intuitionistic fuzzy metric space, Jain et al. [2] discussed the notion of the compatibility of type $(P)$; Sedghi et al. [3] proved some common fixed point theorems for weakly compatible maps using contractive conditions of integral type. The paper [1] is the inspiration of a large number of papers [4-7] that employ the use of modified intuitionistic fuzzy metric space and its applications.

In this paper, we prove some new common fixed point theorems in modified intuitionistic fuzzy metric spaces. While proving our results, we utilize the idea of compatibility due to Jungck [8] together with weakly reciprocal continuity due to Pant et al. [16]. Consequently, our results improve and sharpen many known common fixed point theorems available in the existing literature of modified intuitionistic fuzzy fixed point theory.

Firstly, we recall the following notions that will be used in the sequel.
Lemma 1 (see [10]). Consider the set $L^{*}$ and the operation $\leq_{L^{*}}$ defined by

$$
\begin{array}{r}
L^{*}=\left\{\left(x_{1}, x_{2}\right):\left(x_{1}, x_{2}\right) \in[0,1]^{2}, x_{1}+x_{2} \leq 1\right\} \\
\left(x_{1}, x_{2}\right) \leq_{L^{*}}\left(y_{1}, y_{2}\right) \Longleftrightarrow x_{1} \leq y_{1}, x_{2} \geq y_{2},
\end{array}
$$

for every $\left(x_{1}, x_{2}\right),\left(y_{1}, y_{2}\right)$ in $L^{*}$. Then, $\left(L^{*}, \leq_{L^{*}}\right)$ is a complete lattice.

One denotes its units by $0_{L^{*}}=(0,1)$ and $1_{L^{*}}=(1,0)$.

Definition 2 (see [11]). A triangular norm ( $t$-norm) on $L^{*}$ is a mapping $\mathscr{F}:\left(L^{*}\right)^{2} \rightarrow L^{*}$ satisfying the following conditions:

(1) $\mathscr{F}\left(x, 1_{L^{*}}\right)=x$ for all $x$ in $L^{*}$,

(2) $\mathscr{F}(x, y)=\mathscr{F}(y, x)$ for all $x, y$ in $L^{*}$,

(3) $\mathscr{F}(x, \mathscr{F}(y, z))=\mathscr{F}(\mathscr{F}(x, y), z)$ for all $x, y, z$ in $L^{*}$,

(4) if for all $x, x^{\prime}, y, y^{\prime}$ in $L^{*}, x \leq_{L^{*}} x^{\prime}$ and $y \leq_{L^{*}} y^{\prime}$ imply $\mathscr{F}(x, y) \leq_{L^{*}} \mathscr{F}\left(x^{\prime}, y^{\prime}\right)$.

Definition 3 (see $[10,11])$. A continuous $t$-norm $\mathscr{F}$ on $L^{*}$ is called continuous $t$-representable if and only if there exist a 
continuous $t$-norm $*$ and a continuous $t$-conorm $\diamond$ on $[0,1]$ such that for all $x=\left(x_{1}, x_{2}\right), y=\left(y_{1}, y_{2}\right) \in L^{*}[0,1]^{2}$, $\mathscr{F}(x, y)=\left(x_{1} * y_{1}, x_{2} \diamond y_{2}\right)$.

Definition 4 (see [1]). Let $M, N$ are fuzzy sets from $X^{2} \times$ $(0,+\infty) \rightarrow[0,1]$ such that $M(x, y, t)+N(x, y, t) \leq 1$ for all $x, y$ in $X$ and $t>0$. The 3 -tuple $\left(X, \zeta_{M, N}, \mathscr{F}\right)$ is said to be a modified intuitionistic fuzzy metric space if $X$ is an arbitrary nonempty set, $\mathscr{F}$ is a continuous $t$-representable, and $\zeta_{M, N}$ is a mapping $X^{2} \times(0,+\infty) \rightarrow L^{*}$ satisfying the following conditions for every $x, y$ in $X$ and $t, s>0$ :
(a) $\zeta_{M, N}(x, y, t)>_{L^{*}} 0_{L^{*}}$
(b) $\zeta_{M, N}(x, y, t)=1_{L^{*}}$ if and only if $x=y$,
(c) $\zeta_{M, N}(x, y, t)=\zeta_{M, N}(y, x, t)$,
(d) $\zeta_{M, N}(x, y, t+s) \geq_{L^{*}} \mathscr{F}\left(\zeta_{M, N}(x, z, t), \zeta_{M, N}(z, y, s)\right)$,
(e) $\zeta_{M, N}(x, y, \cdot):(0,+\infty) \rightarrow L^{*}$ is continuous.

In this case, $\zeta_{M, N}$ is called a modified intuitionistic fuzzy metric. Here, $\zeta_{M, N}(x, y, t)=(M(x, y, t), N(x, y, t))$.

Remark 5 (see [12]). In a modified intuitionistic fuzzy metric space $\left(X, \zeta_{M, N}, \mathscr{F}\right), M(x, y, \cdot)$ is nondecreasing, and $N(x, y, \cdot)$ is nonincreasing for all $x, y$ in $X$. Hence, $\zeta_{M, N}$ $(x, y, t)$ is nondecreasing with respect to $t$ for all $x, y$ in $X$.

Definition 6 (see [1]). A sequence $\left\{x_{n}\right\}$ in a modified intuitionistic fuzzy metric space $\left(X, \zeta_{M, N}, \mathscr{F}\right)$ is called a Cauchy sequence if for each $\varepsilon>0$ and $t>0$, there exists $n_{0} \in \mathbb{N}$ such that $\zeta_{M, N}\left(x_{n}, x_{m}, t\right)>_{L^{*}}(1-\varepsilon, \varepsilon)$ for each $n, m \geq n_{0}$ and for all $t$.

Definition 7 (see [1]). A sequence $\left\{x_{n}\right\}$ in a modified intuitionistic fuzzy metric space $\left(X, \zeta_{M, N}, \mathscr{F}\right)$ is said to be convergent to $x$ in $X$, denoted by $x_{n} \rightarrow x$ if $\lim _{n \rightarrow \infty} \zeta_{M, N}\left(x_{n}, x, t\right)=$ $1_{L^{*}}$ for all $t$.

Definition 8 (see [1]). A modified intuitionistic fuzzy metric space $\left(X, \zeta_{M, N}, \mathscr{F}\right)$ is said to be complete if and only if every Cauchy sequence is convergent to a point of it.

Definition 9 (see $[1,13]$ ). A pair of self-mappings $(f, g)$ of modified intuitionistic fuzzy metric space $\left(X, \zeta_{M, N}, \mathscr{F}\right)$ is said to be compatible if $\lim _{n \rightarrow \infty} \zeta_{M, N}\left(f g x_{n}, g f x_{n}, t\right)=1_{L^{*}}$ whenever $\left\{x_{n}\right\}$ is a sequence in $X$ such that $\lim _{n \rightarrow \infty} f\left(x_{n}\right)=$ $\lim _{n \rightarrow \infty} g\left(x_{n}\right)=z$ for some $z$ in $X$.

Definition 10 (see [13]). Two self-mappings $f$ and $g$ are called noncompatible if there exists at least one sequence $\left\{x_{n}\right\}$ such that $\lim _{n \rightarrow \infty} f\left(x_{n}\right)=\lim _{n \rightarrow \infty} g\left(x_{n}\right)=z$ for some $z$ in $X$ but either $\lim _{n \rightarrow \infty} \zeta_{M, N}\left(f g x_{n}, g f x_{n}, t\right) \neq 1_{L^{*}}$ or the limit does not exist for all $z$ in $X$.

Definition 11 (see $[14,15])$. A pair of self mappings $(f, g)$ of a modified intuitionistic fuzzy metric space $\left(X, \zeta_{M, N}, \mathscr{F}\right)$ is said to be $R$-weakly commuting at a point $x$ in $X$ if $\zeta_{M, N}\left(f g x_{n}, g f x_{n}, t\right) \geq_{L^{*}} \zeta_{M, N}\left(f x_{n}, g x_{n}, t / R\right)$ for some $R>0$.
Definition 12 (see $[14,15]$ ). The two self-maps $f$ and $g$ of a modified intuitionistic fuzzy metric space $\left(X, \zeta_{M, N}\right.$, $\mathscr{F})$ are called pointwise $R$-weakly commuting on $X$ if given $x$ in $X$, there exists $R>0$ such that $\zeta_{M, N}$ $(f g x, g f x, t) \geq_{L^{*}} \zeta_{M, N}(f x, g x, t / R)$.

Definition 13 (see [15]). The two self-maps $f$ and $g$ of a modified intuitionistic fuzzy metric space $\left(X, \zeta_{M, N}, \mathscr{F}\right)$ are called $R$-weakly commuting of type $\left(A_{g}\right)$ if there exists some $R>0$ such that $\zeta_{M, N}(f f x, g f x, t) \geq \zeta_{M, N}(f x, g x, t / R)$ for all $x$ in $X$. Similarly, two self-mappings $f$ and $g$ of a modified intuitionistic fuzzy metric space $\left(X, \zeta_{M, N}, \mathscr{F}\right)$ are called $R$ weakly commuting of type $\left(A_{f}\right)$ if there exists some $R>0$ such that $\zeta_{M, N}(f g x, g g x, t) \geq_{L^{*}} \zeta_{M, N}(f x, g x, t / R)$ for all $x$ in $X$.

It is obvious that pointwise $R$-weakly commuting maps commute at their coincidence points and pointwise $R$-weak commutativity is equivalent to commutativity at coincidence points. It may be noted that both compatible and noncompatible mappings can be $R$-weakly commuting of type $\left(A_{g}\right)$ or $\left(A_{f}\right)$, but the converse needs not be true.

Definition 14 (see [2]). Two self-mappings $f$ and $g$ of a modified intuitionistic fuzzy metric space $\left(X, \zeta_{M, N}, \mathscr{F}\right)$ are called $R$-weakly commuting of type $(P)$ if there exists some $R>0$ such that $\zeta_{M, N}(f f x, g g x, t) \geq_{L^{*}} \zeta_{M, N}(f x, g x, t / R)$ for all $x$ in $X$.

In 1999, Pant [9] introduced a new continuity condition, known as reciprocal continuity as follows.

Definition 15 (see [9]). Two self-mappings $f$ and $g$ are called reciprocally continuous if $\lim _{n \rightarrow \infty} f g x_{n}=f z$ and $\lim _{n \rightarrow \infty} g f x_{n}=g z$, whenever $\left\{x_{n}\right\}$ is a sequence such that $\lim _{n \rightarrow \infty} f x_{n}=\lim _{n \rightarrow \infty} g x_{n}=z$ for some $z$ in $X$.

If $f$ and $g$ are both continuous, then they are obviously reciprocally continuous, but the converse is not true.

Recently, Pant et al. [16] generalized the notion of reciprocal continuity to weak reciprocal continuity as follows.

Definition 16 (see [16]). Two self-mappings $f$ and $g$ are called weakly reciprocally continuous if $\lim _{n \rightarrow \infty} f g x_{n}=f z$ or $\lim _{n \rightarrow \infty} g f x_{n}=g z$ whenever $\left\{x_{n}\right\}$ is a sequence such that $\lim _{n \rightarrow \infty} f x_{n}=\lim _{n \rightarrow \infty} g x_{n}=z$ for some $z$ in $X$.

If $f$ and $g$ are reciprocally continuous, then they are obviously weak reciprocally continuous, but the converse is not true. Now, with an application of weak reciprocal continuity, we prove common fixed point theorems under contractive conditions that extend the scope of the study of common fixed point theorems from the class of compatible continuous mappings to a wider class of mappings which also includes noncompatible mappings.

\section{Lemmas}

The proof of our result is based upon the following lemmas. 
Lemma 17 (see [2]). Let $\left(X, \zeta_{M, N}, \mathscr{T}\right)$ be a modified intuitionistic fuzzy metric space and for all $x, y \in X, t>0$ and if for $a$ number $k \in(0,1)$,

$$
\zeta_{M, N}(x, y, k t) \geq_{L^{*}} \zeta_{M, N}(x, y, t) .
$$

Then $x=y$.

Lemma 18 (see [2]). Let $\left(X, \zeta_{M, N}, \mathscr{T}\right)$ be a modified intuitionistic fuzzy metric space and $\left\{y_{n}\right\}$ a sequence in $X$. If there exist a number $k \in(0,1)$ such that

$$
\begin{aligned}
& \zeta_{M, N}\left(y_{n}, y_{n+1}, k t\right) \\
& \quad \geq_{L^{*}} \zeta_{M, N}\left(y_{n-1}, y_{n}, t\right) \quad \forall t>0, n=1,2,3, \ldots,
\end{aligned}
$$

then $\left\{y_{n}\right\}$ is a Cauchy sequence in $X$.

\section{Main Results}

3.1. Section I: Pointwise R-weakly Commuting Pairs and Fixed Point

Lemma 19. Let $\left(X, \zeta_{M, N}, \mathscr{T}\right)$ be a modified intuitionistic fuzzy metric space, and let $(A, S)$ and $(B, T)$ be pairs of self-mappings on $X$ satisfying

$$
A(X) \subseteq T(X), B(X) \subseteq S(X),
$$

there exists a constant $k \in(0,1)$ such that

$$
\begin{aligned}
& \zeta_{M, N}(A x, B y, k t) \\
& \geq_{L^{*}} \min \{ \zeta_{M, N}(T y, B y, t), \\
& \zeta_{M, N}(S x, A x, t), \\
& \zeta_{M, N}(S x, B y, \alpha t), \\
& \zeta_{M, N}(T y, A x,(2-\alpha) t), \\
&\left.\zeta_{M, N}(T y, S x, t)\right\}
\end{aligned}
$$

for all $x, y \in X, t>0$ and $\alpha \in(0,2)$. Then, the continuity of one of the mappings in compatible pair $(A, S)$ or $(B, T)$ on $\left(X, \zeta_{M, N}, \mathscr{T}\right)$ implies their reciprocal continuity.

Proof. First, assume that $A$ and $S$ are compatible and $S$ is continuous. We show that $A$ and $S$ are reciprocally continuous. Let $\left\{u_{n}\right\}$ be a sequence such that $A u_{n} \rightarrow z$ and $S u_{n} \rightarrow z$ for some $z$ in $X$ as $n \rightarrow \infty$. Since $S$ is continuous, we have $S A u_{n} \rightarrow S z$ and $S S u_{n} \rightarrow S z$ as $n \rightarrow \infty$ and since $(A, S)$ is compatible, we have for all $t>0$,

$$
\begin{gathered}
\lim _{n \rightarrow \infty} \zeta_{M, N}\left(A S u_{n}, S A u_{n}, t\right)=1_{L^{*}}, \\
\lim _{n \rightarrow \infty} \zeta_{M, N}\left(A S u_{n}, S z, t\right)=1_{L^{*}} .
\end{gathered}
$$

That is, $A S u_{n} \rightarrow S z$ as $n \rightarrow \infty$. By (4), for each $n$, there exists $v_{n}$ in $X$ such that $A S u_{n}=T v_{n}$. Thus, we have $S S u_{n} \rightarrow S z$, $S A u_{n} \rightarrow S z, A S u_{n} \rightarrow S z$, and $T v_{n} \rightarrow S z$ as $n \rightarrow \infty$ whenever $A S u_{n}=T v_{n}$.
Now, we claim that $B v_{n} \rightarrow S z$ as $n \rightarrow \infty$. Suppose not, then, by (5), taking $\alpha=1$,

$$
\begin{aligned}
\zeta_{M, N}\left(A S u_{n}, B v_{n}, k t\right) \geq_{L^{*}} \min & \left\{\zeta_{M, N}\left(T v_{n}, B v_{n}, t\right),\right. \\
& \zeta_{M, N}\left(S S u_{n}, A S u_{n}, t\right), \\
& \zeta_{M, N}\left(S S u_{n}, B v_{n}, t\right), \\
& \zeta_{M, N}\left(T v_{n}, A S u_{n}, t\right), \\
& \left.\zeta_{M, N}\left(T v_{n}, S S u_{n}, t\right)\right\} .
\end{aligned}
$$

Taking $n \rightarrow \infty$, we have

$$
\begin{aligned}
\zeta_{M, N}\left(S z, \lim _{n \rightarrow \infty} B v_{n}, k t\right) \\
\geq_{L^{*}} \min \left\{\zeta_{M, N}\left(S z, \lim _{n \rightarrow \infty} B v_{n}, t\right), \zeta_{M, N}(S z, S z, t),\right. \\
\\
\zeta_{M, N}\left(S z, \lim _{n \rightarrow \infty} B v_{n}, t\right), \zeta_{M, N}(S z, S z, t), \\
\left.\zeta_{M, N}(S z, S z, t)\right\} \\
=\min \left\{\zeta_{M, N}\left(S z, \lim _{n \rightarrow \infty} B v_{n}, t\right), 1_{L^{*}},\right. \\
\left.\zeta_{M, N}\left(S z, \lim _{n \rightarrow \infty} B v_{n}, t\right), 1_{L^{*}}, 1_{L^{*}}\right\} \\
=\zeta_{M, N}\left(S z, \lim _{n \rightarrow \infty} B v_{n}, t\right) .
\end{aligned}
$$

By Lemma 17, we have $\lim _{n \rightarrow \infty} B v_{n}=S z$.

Claim that $A z=S z$. Again, by (5), taking $\alpha=1$,

$$
\begin{aligned}
& \zeta_{M, N}\left(A z, B v_{n}, k t\right) \\
& \geq_{L^{*}} \min \left\{\zeta_{M, N}\left(T v_{n}, B v_{n}, t\right), \zeta_{M, N}(S z, A z, t),\right. \\
& \zeta_{M, N}\left(S z, B v_{n}, t\right), \\
&\left.\zeta_{M, N}\left(T v_{n}, A z, t\right), \zeta_{M, N}\left(T v_{n}, S z, t\right)\right\} .
\end{aligned}
$$

Taking $n \rightarrow \infty$, we get

$$
\begin{aligned}
\zeta_{M, N}(A z, S z, k t) \geq L_{L^{*}} \min \left\{\zeta_{M, N}(S z, S z, t), \zeta_{M, N}(S z, A z, t),\right. \\
\zeta_{M, N}(S z, S z, t), \\
\left.\zeta_{M, N}(S z, A z, t), \zeta_{M, N}(S z, S z, t)\right\} \\
=\min \left\{1_{L^{*}}, \zeta_{M, N}(S z, A z, t), 1_{L^{*}},\right. \\
\left.\quad \zeta_{M, N}(S z, A z, t), 1_{L^{*}}\right\} \\
=\zeta_{M, N}(S z, A z, t) .
\end{aligned}
$$

By Lemma 17, $A z=S z$. 
Therefore, $S A u_{n} \rightarrow S z, A S u_{n} \rightarrow S z=A z$ as $n \rightarrow \infty$.

Hence, $A$ and $S$ are reciprocally continuous on $X$. If the pair $(B, T)$ is assumed to be compatible and $T$ is continuous, the proof is similar.

Theorem 20. Let $\left(X, \zeta_{M, N}, \mathscr{T}\right)$ be a complete modified intuitionistic fuzzy metric space. Further, let $(A, S)$ and $(B, T)$ be pointwise $R$-weakly commuting pairs of self-mappings of $X$ satisfying (4), (5). If one of the mappings in compatible pair $(A, S)$ or $(B, T)$ is continuous, then $A, B, S$, and T have a unique common fixed point in $X$.

Proof. Let $x_{0} \in X$. By (4), we define the sequences $\left\{x_{n}\right\}$ and $\left\{y_{n}\right\}$ in $X$ such that for all $n=0,1,2, \ldots$,

$$
y_{2 n}=A x_{2 n}=T x_{2 n+1}, \quad y_{2 n+1}=B x_{2 n+1}=S x_{2 n+2} .
$$

We show that $\left\{y_{n}\right\}$ is a Cauchy sequence in $X$. By (5) taking $\alpha=1-\beta, \beta \in(0,1)$, we have

$$
\begin{aligned}
& \zeta_{M, N}\left(y_{2 n+1}, y_{2 n+2}, k t\right) \\
&= \zeta_{M, N}\left(B x_{2 n+1}, A x_{2 n+2}, k t\right) \\
&= \zeta_{M, N}\left(A x_{2 n+2}, B x_{2 n+1}, k t\right) \\
& \geq_{L^{*}} \min \left\{\zeta_{M, N}\left(T x_{2 n+1}, B x_{2 n+1}, t\right),\right. \\
& \zeta_{M, N}\left(S x_{2 n+2}, A x_{2 n+2}, t\right), \\
& \zeta_{M, N}\left(S x_{2 n+2}, B x_{2 n+1},(1-\beta) t\right), \\
& \zeta_{M, N}\left(T x_{2 n+1}, A x_{2 n+2},(1+\beta) t\right), \\
&\left.\zeta_{M, N}\left(T x_{2 n+1}, S x_{2 n+2}, t\right)\right\} \\
&=\min \{ \zeta_{M, N}\left(y_{2 n}, y_{2 n+1}, t\right), \zeta_{M, N}\left(y_{2 n+1}, y_{2 n+2}, t\right), \\
& \zeta_{M, N}\left(y_{2 n+1}, y_{2 n+1},(1-\beta) t\right), \\
& \zeta_{M, N}\left(y_{2 n}, y_{2 n+2},(1+\beta) t\right), \\
&\left.\zeta_{M, N}\left(y_{2 n}, y_{2 n+1}, t\right)\right\} \\
&=\min \{ \zeta_{M, N}\left(y_{2 n}, y_{2 n+1}, t\right), \zeta_{M, N}\left(y_{2 n+1}, y_{2 n+2}, t\right), \\
& \geq_{L^{*}}, \zeta_{M, N}\left(y_{2 n}, y_{2 n+1}, t\right), \\
& \zeta_{M, N}\left(y_{2 n+1}, y_{2 n+2}, \beta t\right), \\
&\left.\zeta_{M, N}\left(y_{2 n}, y_{2 n+1}, t\right)\right\} \\
& \min \left\{\zeta_{M, N}\left(y_{2 n}, y_{2 n+1}, t\right), \zeta_{M, N}\left(y_{2 n+1}, y_{2 n+2}, t\right),\right. \\
&\left.\zeta_{M, N}\left(y_{2 n+1}, y_{2 n+2}, \beta t\right)\right\} .
\end{aligned}
$$

Taking $\beta \rightarrow 1$, we have

$$
\begin{aligned}
\zeta_{M, N}\left(y_{2 n+1}, y_{2 n+2}, k t\right) \\
\geq_{L^{*}} \min \left\{\zeta_{M, N}\left(y_{2 n}, y_{2 n+1}, t\right),\right. \\
\zeta_{M, N}\left(y_{2 n+1}, y_{2 n+2}, t\right), \\
\left.\zeta_{M, N}\left(y_{2 n+1}, y_{2 n+2}, t\right)\right\}, \\
\zeta_{M, N}\left(y_{2 n+1}, y_{2 n+2}, k t\right) \\
\geq_{L^{*}} \min \left\{\zeta_{M, N}\left(y_{2 n}, y_{2 n+1}, t\right), \zeta_{M, N}\left(y_{2 n+1}, y_{2 n+2}, t\right)\right\} \\
\zeta_{M, N}\left(y_{2 n+1}, y_{2 n+2}, k t\right) \geq_{L^{*}} \zeta_{M, N}\left(y_{2 n}, y_{2 n+1}, t\right) .
\end{aligned}
$$

Similarly,

$$
\zeta_{M, N}\left(y_{2 n+2}, y_{2 n+3}, k t\right) \geq_{L^{*}} \zeta_{M, N}\left(y_{2 n+1}, y_{2 n+2}, t\right) .
$$

Therefore, for any $n$ and $t$, we have

$$
\zeta_{M, N}\left(y_{n}, y_{n+1}, k t\right) \geq_{L^{*}} \zeta_{M, N}\left(y_{n-1}, y_{n}, t\right) .
$$

Hence, by Lemma $18,\left\{y_{n}\right\}$ is a Cauchy sequence in $X$. Since $X$ is complete, $\left\{y_{n}\right\}$ converges to $z$ in $X$. Its subsequences $\left\{A x_{2 n}\right\}$, $\left\{T x_{2 n+1}\right\},\left\{B x_{2 n+1}\right\}$, and $\left\{S x_{2 n+2}\right\}$ also converge to $z$.

Now, suppose that $(A, S)$ is a compatible pair and $S$ is continuous. Then, by Lemma $17, A$ and $S$ are reciprocally continuous, then $S A x_{n} \rightarrow S z, A S x_{n} \rightarrow A z$ as $n \rightarrow \infty$. As, $(A, S)$, is a compatible pair. This implies that

$$
\begin{gathered}
\lim _{n \rightarrow \infty} \zeta_{M, N}\left(A S x_{n}, S A x_{n}, t\right)=1_{L^{*}}, \\
\zeta_{M, N}(A z, S z, t)=1_{L^{*}} .
\end{gathered}
$$

Hence, $A z=S z$.

Since $A(X) \subseteq T(X)$, there exists a point $p$ in $X$ such that $A z=T p=S z$.

By (5), taking $\alpha=1$,

$$
\begin{gathered}
\zeta_{M, N}(A z, B p, k t) \\
\geq_{L^{*}} \min \left\{\zeta_{M, N}(T p, B p, t), \zeta_{M, N}(S z, A z, t),\right. \\
\zeta_{M, N}(S z, B p, t), \zeta_{M, N}(T p, A z, t), \\
\left.\zeta_{M, N}(T p, S z, t)\right\}, \\
\zeta_{M, N}(A z, B p, k t) \\
\geq_{L^{*}} \min \left\{\zeta_{M, N}(A z, B p, t), \zeta_{M, N}(A z, A z, t),\right. \\
\zeta_{M, N}(A z, B p, t), \zeta_{M, N}(A z, A z, t), \\
\left.\zeta_{M, N}(A z, A z, t)\right\} . \\
\zeta_{M, N}(A z, B p, k t) \geq_{L^{*}} \zeta_{M, N}(A z, B p, t) .
\end{gathered}
$$

Thus, by Lemma 17, we have $A z=B p$.

Thus, $A z=B p=S z=T p$. 
Since $A$ and $S$ are pointwise $R$-weakly commuting mappings, there exists $R>0$, such that

$$
\zeta_{M, N}(A S z, S A z, t) \geq_{L^{*}} \zeta_{M, N}\left(A z, S z, \frac{t}{R}\right)=1_{L^{*}}
$$

Therefore, $A S z=S A z$ and $A A z=A S z=S A z=S S z$.

Similarly, since $B$ and $T$ are pointwise $R$-weakly commuting mappings, we have $B B p=B T p=T B p=T T p$.

Again, by (5), taking $\alpha=1$,

$$
\begin{aligned}
& \zeta_{M, N}(A A z, B p, k t) \\
& \geq_{L^{*}} \min \left\{\zeta_{M, N}(T p, B p, t), \zeta_{M, N}(S A z, A A z, t),\right. \\
& \zeta_{M, N}(S A z, B p, t), \\
& \zeta_{M, N}(T p, A A z, t), \\
&\left.\zeta_{M, N}(T p, S A z, t)\right\}, \\
& \zeta_{M, N}(A A z, A z, k t) \\
& \geq_{L^{*}} \min \left\{\zeta_{M, N}(T p, T p, t), \zeta_{M, N}(A A z, A A z, t),\right. \\
& \zeta_{M, N}(A A z, A z, t), \\
& \zeta_{M, N}(A z, A A z, t), \\
&\left.\zeta_{M, N}(A z, A A z, t)\right\}, \\
& \zeta_{M, N}(A A z, A z, k t) \geq_{L^{*}} \zeta_{M, N}(A A z, A z, t) .
\end{aligned}
$$

By Lemma 17, we have $A A z=A z=S A z$. Hence $A z$ is a common fixed point of $A$ and $S$. Similarly, by (5), $B p=A z$ is a common fixed point of $B$ and T. Hence, $A z$ is a common fixed point of $A, B, S$, and $T$.

For Uniqueness. Suppose that $A p(\neq A z)$ is another common fixed point of $A, B, S$, and $T$. Then, by (5), taking $\alpha=1$,

$$
\begin{gathered}
\zeta_{M, N}(A A z, B A p, k t) \\
\geq_{L^{*}} \min \left\{\zeta_{M, N}(T A p, B A p, t), \zeta_{M, N}(S A z, A A z, t),\right. \\
\zeta_{M, N}(S A z, B A p, t), \\
\zeta_{M, N}(T A p, A A z, t), \\
M(T A p, S A z, t)\}, \\
\zeta_{M, N}(A z, A p, k t) \\
\geq_{L^{*}} \min \left\{\zeta_{M, N}(A p, A p, t), \zeta_{M, N}(A z, A z, t),\right. \\
\zeta_{M, N}(A z, A p, t), \\
\zeta_{M, N}(A p, A z, t), \\
\left.\zeta_{M, N}(A p, A z, t)\right\} \\
\zeta_{M, N}(A z, A p, k t) \geq_{L^{*}} \zeta_{M, N}(A z, A p, t) .
\end{gathered}
$$

By Lemma 17, $A z=A p$.

Thus, the uniqueness follows.
Corollary 21. Let $\left(X, \zeta_{M, N}, \mathscr{T}\right)$ be a complete modified intuitionistic fuzzy metric space. Further, let $A$ and $B$ be reciprocally continuous mappings on $X$ satisfying

$$
\begin{gathered}
\zeta_{M, N}(A x, B y, k t) \geq_{L^{*}} \min \left\{\zeta_{M, N}(y, B y, t), \zeta_{M, N}(x, A x, t),\right. \\
\zeta_{M, N}(x, B y, \alpha t), \\
\zeta_{M, N}(y, A x,(2-\alpha) t), \\
\left.\zeta_{M, N}(y, x, t)\right\}
\end{gathered}
$$

for all $x, y \in X, t>0$ and $\alpha \in(0,2)$, then pair $A$ and $B$ has $a$ unique common fixed point.

Example 22. Let $X=[2,20]$ and for each $t>0$, define

$$
\zeta_{M, N}(x, y, t)=\left(\frac{t}{t+|x-y|}, \frac{|x-y|}{t+|x-y|}\right) .
$$

Then, $\left(X, \zeta_{M, N}, \mathscr{T}\right)$ is complete modified intuitionistic fuzzy metric space. Let $A, B, S$, and $T$ be self-mappings of $X$ defined as

$$
\begin{gathered}
A(2)=2, \quad A u=3 \quad \text { if } u>2, \\
B(u)=2 \quad \text { if } u=2 \text { or } u>5, \quad B u=6 \quad \text { if } 2<u \leq 5, \\
S(2)=2, \quad S(u)=6 \quad \text { if } u>2, \\
T(2)=2, \quad T(u)=12 \quad \text { if } 2<u \leq 5, \\
T(u)=u-3 \quad \text { if } u>5 .
\end{gathered}
$$

Then, $A, B, S$, and $T$ satisfy all the conditions of the above theorem with $k \in(0,1)$ and have a unique common fixed point $u=2$.

\subsection{Section II: Weak Reciprocal Continuity and Fixed Point Theorem}

Theorem 23. Let $f$ and $g$ be weakly reciprocally continuous self-mappings of a complete modified intuitionistic fuzzy metric space $\left(X, \zeta_{M, N}, \mathcal{T}\right)$ satisfying the following conditions:

$$
f(X) \subset g(X)
$$

for any $x, y \in X, t>0, k \in(0,1)$ such that

$$
\begin{gathered}
\zeta_{M, N}(f x, f y, k t) \\
\geq_{L^{*}} \min \left\{\zeta_{M, N}(g x, g y, t), \zeta_{M, N}(g x, f y, 2 t),\right. \\
\zeta_{M, N}(f x, g x, t), \zeta_{M, N}(f x, g y, t), \\
\left.\zeta_{M, N}(f y, g y, t)\right\} .
\end{gathered}
$$

If $f$ and $g$ are either compatible or $R$-weakly commuting of type $\left(A_{g}\right)$ or $R$-weakly commuting of type $\left(A_{f}\right)$ or $R$-weakly commuting of type $(P)$, then $f$ and $g$ have a unique common fixed point. 
Proof. Let $x_{0}$ be any point in $X$. Then, as $f(X) \subset g(X)$, there exist a sequence of points $\left\{x_{n}\right\}$ such that $f\left(x_{n}\right)=g\left(x_{n+1}\right)$.

Also, define a sequence $\left\{y_{n}\right\}$ in $X$ as

$$
y_{n}=f\left(x_{n}\right)=g\left(x_{n+1}\right) .
$$

Now, we show that $\left\{y_{n}\right\}$ is a Cauchy sequence in $X$. For proving this, by (25), we have

$$
\begin{aligned}
& \zeta_{M, N}\left(y_{n}, y_{n+1}, k t\right) \\
& =\zeta_{M, N}\left(f x_{n}, f x_{n+1}, k t\right) \\
& \geq_{L^{*}} \min \left\{\zeta_{M, N}\left(g x_{n}, g x_{n+1}, t\right),\right. \\
& \zeta_{M, N}\left(g x_{n}, f x_{n+1}, 2 t\right), \\
& \zeta_{M, N}\left(f x_{n}, g x_{n}, t\right) \text {, } \\
& \zeta_{M, N}\left(f x_{n}, g x_{n+1}, t\right), \\
& \left.\zeta_{M, N}\left(f x_{n+1}, g x_{n+1}, t\right)\right\} \\
& =\min \left\{\zeta_{M, N}\left(y_{n-1}, y_{n}, t\right)\right. \text {, } \\
& \zeta_{M, N}\left(y_{n-1}, y_{n+1}, 2 t\right) \text {, } \\
& \zeta_{M, N}\left(y_{n}, y_{n-1}, t\right) \text {, } \\
& \zeta_{M, N}\left(y_{n}, y_{n}, t\right) \text {, } \\
& \left.\zeta_{M, N}\left(y_{n+1}, y_{n}, t\right)\right\} \\
& \geq_{L^{*}} \min \left\{\zeta_{M, N}\left(y_{n-1}, y_{n}, t\right)\right. \text {, } \\
& \zeta_{M, N}\left(y_{n-1}, y_{n}, t\right) \text {, } \\
& \zeta_{M, N}\left(y_{n}, y_{n+1}, t\right) \text {, } \\
& \zeta_{M, N}\left(y_{n}, y_{n-1}, t\right) \text {, } \\
& \left.1, \zeta_{M, N}\left(y_{n+1}, y_{n}, t\right)\right\} \\
& =\min \left\{\zeta_{M, N}\left(y_{n-1}, y_{n}, t\right)\right. \text {, } \\
& \left.\zeta_{M, N}\left(y_{n}, y_{n+1}, t\right)\right\} \\
& \zeta_{M, N}\left(y_{n}, y_{n+1}, k t\right) \geq_{L^{*}} \zeta_{M, N}\left(y_{n-1}, y_{n}, t\right) .
\end{aligned}
$$

Then, by Lemma $18,\left\{y_{n}\right\}$ is a Cauchy sequence in $X$. As $X$ is complete, there exists a point $z$ in $X$ such that $\lim _{n \rightarrow \infty} y_{n}=z$. Therefore, by (26), we have $\lim _{n \rightarrow \infty} y_{n}=\lim _{n \rightarrow \infty} f\left(x_{n}\right)=$ $\lim _{n \rightarrow \infty} g\left(x_{n+1}\right)=z$.

Suppose that $f$ and $g$ are compatible mappings. Now, by weak reciprocal continuity of $f$ and $g$, it implies that $\lim _{n \rightarrow \infty}$ $f g\left(x_{n}\right)=f z$ or $\lim _{n \rightarrow \infty} g f\left(x_{n}\right)=g z$. Let $\lim _{n \rightarrow \infty} g f\left(x_{n}\right)=$ $g z$, then the compatibility of $f$ and $g$ gives

$$
\lim _{n \rightarrow \infty} \zeta_{M, N}\left(f g x_{n}, g f x_{n}, t\right)=1_{L^{*}}
$$

This gives

$$
\lim _{n \rightarrow \infty} \zeta_{M, N}\left(f g x_{n}, g z, t\right)=1_{L^{*}}
$$

Hence, $\lim _{n \rightarrow \infty} f g\left(x_{n}\right)=g z$. By (26), we get $\lim _{n \rightarrow \infty}$ $f g\left(x_{n+1}\right)=\lim _{n \rightarrow \infty} f f\left(x_{n}\right)=g z$.

Therefore, by (25), we get

$$
\begin{array}{r}
\zeta_{M, N}\left(f z, f f x_{n}, k t\right) \geq_{L^{*}} \min \left\{\zeta_{M, N}\left(g z, g f x_{n}, t\right),\right. \\
\zeta_{M, N}\left(g z, f f x_{n}, 2 t\right), \\
\zeta_{M, N}(f z, g z, t), \\
\zeta_{M, N}\left(f z, g f x_{n}, t\right), \\
\left.\zeta_{M, N}\left(f f x_{n}, g f x_{n}, t\right)\right\} .
\end{array}
$$

Taking $n \rightarrow \infty$, we get

$$
\begin{array}{r}
\zeta_{M, N}(f z, g z, k t) \geq_{L^{*}} \min \left\{\zeta_{M, N}(g z, g z, t),\right. \\
\zeta_{M, N}(g z, g z, 2 t), \\
\zeta_{M, N}(f z, g z, t), \\
\zeta_{M, N}(f z, g z, t), \\
\left.\zeta_{M, N}(g z, g z, t)\right\}, \\
\zeta_{M, N}(f z, g z, k t) \geq_{L^{*}} \zeta_{M, N}(f z, g z, t) .
\end{array}
$$

Hence, by Lemma 17, we get $f z=g z$. Again, the compatibility of $f$ and $g$ implies commutativity at a coincidence point. Hence $g f z=f g z=f f z=g g z$. Using (25), we obtain

$$
\begin{gathered}
\zeta_{M, N}(f z, f f z, k t) \\
\geq \geq_{L^{*}} \min \left\{\zeta_{M, N}(g z, g f z, t), \zeta_{M, N}(g z, f f z, 2 t),\right. \\
\zeta_{M, N}(f z, g z, t), \\
\left.\zeta_{M, N}(f z, g f z, t), \zeta_{M, N}(f f z, g f z, t)\right\} \\
=\min \left\{\zeta_{M, N}(f z, f f z, t), \zeta_{M, N}(f z, f f z, 2 t), 1_{L^{*}},\right. \\
\left.\zeta_{M, N}(f z, f f z, t), 1_{L^{*}}\right\} \\
\geq \min \left\{\zeta_{M, N}(f z, f f z, t), \zeta_{M, N}(f z, f f z, t), 1_{L^{*}},\right. \\
\left.\zeta_{M, N}(f z, f f z, t), 1_{L^{*}}\right\}, \\
=\zeta_{M, N}(f z, f f z, t) .
\end{gathered}
$$

That is, $f z=f f z$. Hence, $f z=f f z=g f z$ and $f z$ is a common fixed point of $f$ and $g$.

Next, suppose that $\lim _{n \rightarrow \infty} f g\left(x_{n}\right)=f z$. Then, $f(X) \subset$ $g(X)$ implies that $f z=g u$ for some $u \in X$ and, therefore, $\lim _{n \rightarrow \infty} f g\left(x_{n}\right)=g u$.

The compatibility of $f$ and $g$ implies that $\lim _{n \rightarrow \infty} g f\left(x_{n}\right)=g u$. By the virtue of (26), this gives

$$
\lim _{n \rightarrow \infty} f g\left(x_{n+1}\right)=\lim _{n \rightarrow \infty} f f\left(x_{n}\right)=g u \text {. }
$$


Using (25), we get

$$
\begin{aligned}
\zeta_{M, N}\left(f u, f f x_{n}, k t\right) \geq_{L^{*}} \min & \left\{\zeta_{M, N}\left(g u, g f x_{n}, t\right),\right. \\
& \zeta_{M, N}\left(g u, f f x_{n}, 2 t\right), \\
& \zeta_{M, N}(f u, g u, t), \\
& \zeta_{M, N}\left(f u, g f x_{n}, t\right), \\
& \left.\zeta_{M, N}\left(f f x_{n}, g f x_{n}, t\right)\right\} .
\end{aligned}
$$

Taking $n \rightarrow \infty$, we have

$$
\begin{aligned}
& \zeta_{M, N}(f u, g u, k t) \\
& \geq_{L^{*}} \min \left\{\zeta_{M, N}(g u, g u, t), \zeta_{M, N}(g u, g u, 2 t),\right. \\
& \zeta_{M, N}(f u, g u, t), \\
&\left.\zeta_{M, N}(f u, g u, t), \zeta_{M, N}(g u, g u, t)\right\}, \\
& \zeta_{M, N}(f u, g u, k t) \geq_{L^{*}} \zeta_{M, N}(f u, g u, t) .
\end{aligned}
$$

By Lemma 17, we get $f u=g u$. The compatibility of $f$ and $g$ yields $f g u=g g u=f f u=g f u$. Finally, using (25), we obtain

$$
\begin{gathered}
\zeta_{M, N}(f u, f f u, k t) \\
\geq_{L^{*}} \min \left\{\zeta_{M, N}(g u, g f u, t), \zeta_{M, N}(g u, f f u, 2 t),\right. \\
\zeta_{M, N}(f u, g u, t), \\
\left.\zeta_{M, N}(f u, g f u, t), \zeta_{M, N}(f f u, g f u, t)\right\} \\
=\min \left\{\zeta_{M, N}(f u, f f u, t), \zeta_{M, N}(f u, f f u, 2 t),\right. \\
\left.1_{L^{*}}, \zeta_{M, N}(f u, f f u, t), 1_{L^{*}}\right\} \\
\geq_{L^{*}} \min \left\{\zeta_{M, N}(f u, f f u, t), \zeta_{M, N}(f u, f f u, t),\right. \\
\left.1_{L^{*}}, \zeta_{M, N}(f u, f f u, t), 1_{L^{*}}\right\} \\
=\zeta_{M, N}(f u, f f u, t) .
\end{gathered}
$$

That is, $f u=f f u$. Hence $f u=f f u=g f u$ and $f u$ is a common fixed point of $f$ and $g$.

Now, suppose that $f$ and $g$ are $R$-weakly commuting of type $\left(A_{g}\right)$. Now, weak reciprocal continuity of $f$ and $g$ implies that $\lim _{n \rightarrow \infty} f g\left(x_{n}\right)=f z$ or $\lim _{n \rightarrow \infty} g f\left(x_{n}\right)=g z$. Let us first assume that $\lim _{n \rightarrow \infty} g f\left(x_{n}\right)=g z$. Then, $R$-weak commutativity of type $\left(A_{g}\right)$ of $f$ and $g$ yields

$$
\begin{gathered}
\zeta_{M, N}\left(f f x_{n}, g f x_{n}, t\right) \geq_{L^{*}} \zeta_{M, N}\left(f x_{n}, g x_{n}, \frac{t}{R}\right), \\
\lim _{n \rightarrow \infty} \zeta_{M, N}\left(f f x_{n}, g z, t\right) \geq_{L^{*}} \zeta_{M, N}\left(z, z, \frac{t}{R}\right)=1_{L^{*}} .
\end{gathered}
$$

This gives $\lim _{n \rightarrow \infty} f f x_{n}=g z$. Also, using (25), we get

$$
\begin{gathered}
\zeta_{M, N}\left(f z, f f x_{n}, k t\right) \\
\geq_{L^{*}} \min \left\{\zeta_{M, N}\left(g z, g f x_{n}, t\right), \zeta_{M, N}\left(g z, f f x_{n}, 2 t\right),\right. \\
\zeta_{M, N}(f z, g z, t), \zeta_{M, N}\left(f z, g f x_{n}, t\right), \\
\left.\zeta_{M, N}\left(f f x_{n}, g f x_{n}, t\right)\right\} .
\end{gathered}
$$

Taking $n \rightarrow \infty$, we have

$$
\begin{gathered}
\zeta_{M, N}(f z, g z, k t) \\
\geq_{L^{*}} \min \left\{\zeta_{M, N}(g z, g z, t), \zeta_{M, N}(g z, g z, 2 t),\right. \\
\zeta_{M, N}(f z, g z, t), \\
\left.\zeta_{M, N}(f z, g z, t), \zeta_{M, N}(g z, g z, t)\right\}, \\
\zeta_{M, N}(f z, g z, k t) \geq_{L^{*}} \zeta_{M, N}(f z, g z, t) .
\end{gathered}
$$

Hence, by Lemma 17 , we get $f z=g z$. Again, by using $R$-weak commutativity of type $\left(A_{g}\right)$,

$$
\zeta_{M, N}(f f z, g f z, t) \geq_{L^{*}} \zeta_{M, N}\left(f z, g z, \frac{t}{R}\right)=1_{L^{*}} .
$$

This yields $f f z=g f z$. Therefore, $f f z=f g z=g f z=$ ggz. Using (25), we get

$$
\begin{aligned}
& \zeta_{M, N}(f z, f f z, k t) \\
& \geq_{L^{*}} \min \left\{\zeta_{M, N}(g z, g f z, t), \zeta_{M, N}(g z, f f z, 2 t),\right. \\
& \zeta_{M, N}(f z, g z, t), \\
&\left.\zeta_{M, N}(f z, g f z, t), \zeta_{M, N}(f f z, g f z, t)\right\} \\
&= \min \left\{\zeta_{M, N}(f z, f f z, t), \zeta_{M, N}(f z, f f z, 2 t), 1_{L^{*}},\right. \\
&\left.\zeta_{M, N}(f z, f f z, t), 1_{L^{*}}\right\} \\
& \geq_{L^{*}} \min \left\{\zeta_{M, N}(f z, f f z, t), \zeta_{M, N}(f z, f f z, t), 1_{L^{*}},\right. \\
&\left.\zeta_{M, N}(f z, f f z, t), 1_{L^{*}}\right\} \\
&=\zeta_{M, N}(f z, f f z, t) .
\end{aligned}
$$

That is, $f z=f f z$. Hence, $f z=f f z=g f z$ and $f z$ is a common fixed point of $f$ and $g$.

Similarly, we prove if $\lim _{n \rightarrow \infty} f g\left(x_{n}\right)=f z$.

Suppose that $f$ and $g$ are $R$-weakly commuting of type $\left(A_{f}\right)$. Again, as done above, we can easily prove that $f z$ is a common fixed point of $f$ and $g$.

Finally, suppose that $f$ and $g$ are $R$-weakly commuting of type $(P)$. Weak reciprocal continuity of $f$ and $g$ implies that $\lim _{n \rightarrow \infty} f g\left(x_{n}\right)=f z$ or $\lim _{n \rightarrow \infty} g f\left(x_{n}\right)=g z$. Let us assume that $\lim _{n \rightarrow \infty} g f\left(x_{n}\right)=g z$. Then, $R$-weak commutativity of type $(P)$ of $f$ and $g$ yields

$$
\zeta_{M, N}\left(f f x_{n}, g g x_{n}, t\right) \geq_{L^{*}} \zeta_{M, N}\left(f x_{n}, g x_{n}, \frac{t}{R}\right) .
$$

Taking limit as $n \longrightarrow \infty$,

$$
\lim _{n \rightarrow \infty} \zeta_{M, N}\left(f f x_{n}, g g x_{n}, t\right) \geq_{L^{*}} \zeta_{M, N}\left(z, z, \frac{t}{R}\right)=1_{L^{*}} .
$$

This gives $\lim _{n \rightarrow \infty} \zeta_{M, N}\left(f f x_{n}, g g x_{n}, t\right)=1_{L^{*}}$. 
Using (24) and (26), we have $g f x_{n-1}=g g x_{n} \rightarrow g z$ as $n \rightarrow \infty$; this gives $f f x_{n} \rightarrow g z$ as $n \rightarrow \infty$. Also, using (25) we get

$$
\begin{gathered}
\zeta_{M, N}\left(f z, f f x_{n}, k t\right) \\
\geq_{L^{*}} \min \left\{\zeta_{M, N}\left(g z, g f x_{n}, t\right), \zeta_{M, N}\left(g z, f f x_{n}, 2 t\right),\right. \\
\zeta_{M, N}(f z, g z, t), \zeta_{M, N}\left(f z, g f x_{n}, t\right), \\
\left.\zeta_{M, N}\left(f f x_{n}, g f x_{n}, t\right)\right\} .
\end{gathered}
$$

Taking $n \rightarrow \infty$, we have

$$
\begin{gathered}
\zeta_{M, N}(f z, g z, k t) \\
\geq_{L^{*}} \min \left\{\zeta_{M, N}(g z, g z, t), \zeta_{M, N}(g z, g z, 2 t),\right. \\
\zeta_{M, N}(f z, g z, t), \\
\left.\zeta_{M, N}(f z, g z, t), \zeta_{M, N}(g z, g z, t)\right\}, \\
\zeta_{M, N}(f z, g z, k t) \\
\geq_{L^{*}} \min \left\{1_{L^{*}}, 1_{L^{*}}, \zeta_{M, N}(f z, g z, t),\right. \\
\left.\zeta_{M, N}(f z, g z, t), 1_{L^{*}}\right\}, \\
\zeta_{M, N}(f z, g z, k t) \geq_{L^{*}} \zeta_{M, N}(f z, g z, t) .
\end{gathered}
$$

By Lemma 17, we get $f z=g z$. Again, by using $R$-weak commutativity of type $(P)$,

$$
\zeta_{M, N}(f f z, g g z, t) \geq_{L^{*}} \zeta_{M, N}\left(f z, g z, \frac{t}{R}\right)=1_{L^{*}}
$$

This yields $f f z=g g z$.

Therefore, $f f z=f g z=g f z=g g z$. Using (25), we get

$$
\begin{array}{r}
\zeta_{M, N}(f z, f f z, k t) \\
\geq_{L^{*}} \min \left\{\zeta_{M, N}(g z, g f z, t), \zeta_{M, N}(g z, f f z, 2 t),\right. \\
\zeta_{M, N}(f z, g z, t), \\
\left.\zeta_{M, N}(f z, g f z, t), \zeta_{M, N}(f f z, g f z, t)\right\} \\
=\min \left\{\zeta_{M, N}(f z, f f z, t), \zeta_{M, N}(f z, f f z, 2 t),\right. \\
\zeta_{M, N}(f z, f z, t), \\
\left.\zeta_{M, N}(f z, f f z, t), \zeta_{M, N}(f f z, f f z, t)\right\} \\
\geq_{L^{*}} \min \left\{\zeta_{M, N}(f z, f f z, t), \zeta_{M, N}(f z, f f z, t),\right. \\
\zeta_{M, N}(f z, f z, t), \\
\left.\zeta_{M, N}(f z, f f z, t), \zeta_{M, N}(f f z, f f z, t)\right\} \\
=\zeta_{M, N}(f z, f f z, t) .
\end{array}
$$

By Lemma 17, we get $f z=f f z$. Hence, $f z=f f z=g f z$ and $f z$ is a common fixed point of $f$ and $g$.

Similarly, we prove that if $\lim _{n \rightarrow \infty} f g\left(x_{n}\right)=f z$.
Uniqueness of the common fixed point theorem follows easily in each of the three cases by using (5).

We now give an example to illustrate Theorem 23.

Example 24 (see [16]). Let $\left(X, \zeta_{M, N}, \mathscr{T}\right)$ be modified intuitionistic fuzzy metric space, where $X=[2,20]$, as defined in Example 22.

Define $f, g: X \rightarrow X$ by

$$
\begin{gathered}
f x=2 \quad \text { if } x=2 \text { or } x>5, \\
f x=6 \quad \text { if } 2<x \leq 5, \\
g 2=2, \quad g x=11 \quad \text { if } 2<x \leq 5, \\
g x=\frac{(x+1)}{3} \quad \text { if } x>5 .
\end{gathered}
$$

Let $\left\{x_{n}\right\}$ be a sequence in $X$ such that either $x_{n}=2$ or $x_{n}=$ $5+1 / n$ for each $n$.

Then, clearly, $f$ and $g$ satisfy all the conditions of Theorem 23 and have a unique common fixed point at $x=2$.

Theorem 25. Let $f$ and $g$ be weakly reciprocally continuous noncompatible self-mappings of a modified intuitionistic fuzzy metric space $\left(X, \zeta_{M, N}, \mathscr{T}\right)$ satisfying $(24)$ and

$$
\zeta_{M, N}(f x, f y, k t) \geq_{L^{*}} \zeta_{M, N}(g x, g y, t)
$$

for all $k \geq 0$

$$
\begin{aligned}
\zeta_{M, N}\left(f x, f^{2} x, t\right) & \\
>_{L^{*}} \max & \left\{\zeta_{M, N}(g x, g f x, t), \zeta_{M, N}(f x, g x, t),\right. \\
& \zeta_{M, N}\left(f^{2} x, g f x, t\right), \\
& \left.\zeta_{M, N}(f x, g f x, t), \zeta_{M, N}\left(g x, f^{2} x, t\right)\right\},
\end{aligned}
$$

whenever $f x \neq f^{2} x$ for all $x, y \in X$ and $t>0$.

If $f$ and $g$ are $R$-weakly commuting of type $\left(A_{g}\right)$ or $R$ weakly commuting of type $\left(A_{f}\right)$ or $R$-weakly commuting of type $(P)$, then $f$ and $g$ have common fixed point.

Proof. Since $f$ and $g$ are noncompatible maps, there exists a sequence $\left\{x_{n}\right\}$ in $X$ such that $f x_{n} \rightarrow z$ and $g x_{n} \rightarrow$ $z$ for some $z$ in $X$ as $n \rightarrow \infty$ but either $\lim _{n \rightarrow \infty}$ $\zeta_{M, N}\left(f g x_{n}, g f x_{n}, t\right) \neq 1_{L^{*}}$ or the limit does not exist. Since $f(X) \subset g(X)$, for each $\left\{x_{n}\right\}$ there exists $\left\{y_{n}\right\}$, in $X$ such that $f x_{n}=g y_{n}$. Thus, $f x_{n} \rightarrow z, g x_{n} \rightarrow z$ and $g y_{n} \rightarrow z$ as $n \rightarrow \infty$. By the virtue of this and using (48), we obtain

$$
\begin{gathered}
\zeta_{M, N}\left(f x_{n}, f y_{n}, k t\right) \geq_{L^{*}} \zeta_{M, N}\left(g x_{n}, g y_{n}, t\right), \\
\lim _{n \rightarrow \infty} \zeta_{M, N}\left(z, f y_{n}, k t\right) \geq_{L^{*}} \zeta_{M, N}(z, z, t)=1_{L^{*}} .
\end{gathered}
$$

This gives $f y_{n} \rightarrow z$ as $n \rightarrow \infty$. Therefore, we have $f x_{n} \rightarrow$ $z, g x_{n} \rightarrow z, g y_{n} \rightarrow z, f y_{n} \rightarrow z$

Suppose that $f$ and $g$ are $R$-weakly commuting of type $\left(A_{g}\right)$. Then, weak reciprocal continuity of $f$ and $g$ implies that $f g x_{n} \rightarrow f z$ or $g f x_{n} \rightarrow g z$. Similarly, $f g y_{n} \rightarrow f z$ 
or $g f y_{n} \rightarrow g z$. Let us first assume that $g f y_{n} \rightarrow g z$. Then, $R$-weak commutativity of type $\left(A_{g}\right)$ of $f$ and $g$ yields

$$
\begin{gathered}
\zeta_{M, N}\left(f f y_{n}, g f y_{n}, t\right) \geq_{L^{*}} \zeta_{M, N}\left(f y_{n}, g y_{n}, \frac{t}{R}\right), \\
\lim _{n \rightarrow \infty} \zeta_{M, N}\left(f f y_{n}, g z, t\right) \geq_{L^{*}} \zeta_{M, N}\left(z, z, \frac{t}{R}\right)=1_{L^{*}} .
\end{gathered}
$$

This gives $f f y_{n} \rightarrow g z$. Using (48), we get

$$
\begin{gathered}
\zeta_{M, N}\left(f f y_{n}, f z, k t\right) \geq_{L^{*}} \zeta_{M, N}\left(g f y_{n}, g z, t\right) \\
n \longrightarrow \infty \\
\zeta_{M, N}(g z, f z, k t) \geq_{L^{*}} \zeta_{M, N}(g z, g z, t)=1_{L^{*}}
\end{gathered}
$$

This implies that $f z=g z$. Again, by the virtue of $R$-weak commutativity of type $\left(A_{g}\right)$,

$$
\zeta_{M, N}(f f z, g f z, t) \geq_{L^{*}} \zeta_{M, N}\left(f z, g z, \frac{t}{R}\right)=1_{L^{*}}
$$

This yields $f f z=g f z$ and $f f z=f g z=g f z=g g z$. If $f z \neq f f z$, then by using (49), we get

$$
\begin{aligned}
& \zeta_{M, N}\left(f z, f^{2} z, t\right) \\
&>_{L^{*}} \max \left\{\zeta_{M, N}(g z, g f z, t), \zeta_{M, N}(f z, g z, t),\right. \\
& \zeta_{M, N}\left(f^{2} z, g f z, t\right), \\
&\left.\zeta_{M, N}(f z, g f z, t), \zeta_{M, N}\left(g z, f^{2} z, t\right)\right\} \\
&=\max \left\{\zeta_{M, N}(f z, f f z, t), \zeta_{M, N}(f z, g z, t),\right. \\
& \zeta_{M, N}(f f z, g f z, t), \\
&\left.\zeta_{M, N}(f z, f f z, t), \zeta_{M, N}(f z, f f z, t)\right\}, \\
& \zeta_{M, N}\left(f z, f^{2} z, t\right)>_{L^{*}} 1_{L^{*}},
\end{aligned}
$$

which is a contradiction. Hence, $f z=f f z=g f z$ and $f z$ is a common fixed point of $f$ and $g$.

Similarly, we can prove that if $f g y_{n} \rightarrow f z$, then again $f z$ is a common fixed point of $f$ and $g$. Proof is similar if $f$ and $g$ are $R$-weakly commuting of type $\left(A_{f}\right)$ or $(P)$.

We now give an example to illustrate Theorem 25.

Example 26 (see [16]). Let $\left(X, \zeta_{M, N}, \mathscr{T}\right)$ be modified intuitionistic fuzzy metric space as defined in Example 22 and let $\left\{x_{n}\right\}$ be a sequence in $X$ as in Example 22 .

Define $f, g: X \rightarrow X$ by

$$
\begin{gathered}
f x=2 \quad \text { if } x=2 \text { or } x>5, \\
f x=6 \quad \text { if } 2<x \leq 5, \\
g 2=2, \quad g x=11 \quad \text { if } 2<x \leq 5, \\
g x=\frac{(x+1)}{3} \quad \text { if } x>5 .
\end{gathered}
$$

Let $\left\{x_{n}\right\}$ be a sequence in $X$ such that either $x_{n}=2$ or $x_{n}=5+$ $1 / n$ for each $n$. Then, clearly, $f$ and $g$ satisfy all the conditions of Theorem 25 and have a common fixed point at $x=2$.

Theorem 27. Let $f$ and $g$ be weakly reciprocally continuous noncompatible self-mappings of a modified intuitionistic fuzzy metric space $\left(X, \zeta_{M, N}, \mathscr{T}\right)$ satisfying the conditions (24) and

$$
\begin{gathered}
\zeta_{M, N}(f x, f y, k t) \geq_{L^{*}} \zeta_{M, N}(g x, g y, t) \quad \forall k \geq 0, \\
\zeta_{M, N}\left(f x, f^{2} x, t\right)>_{L^{*}} \zeta_{M, N}\left(g x, g^{2} x, t\right),
\end{gathered}
$$

whenever $f x \neq f^{2} x$ for all $x, y \in X$ and $t>0$.

If $f$ and $g$ are $R$-weakly commuting of type $\left(A_{g}\right)$ or $R$ weakly commuting of type $\left(A_{f}\right)$ or $R$-weakly commuting of type $(P)$, then $f$ and $g$ have common fixed point.

Proof. Since $f$ and $g$ are noncompatible maps, there exists a sequence $\left\{x_{n}\right\}$ in $X$ such that $f x_{n} \rightarrow z$ and $g x_{n} \rightarrow z$ for some $z$ in $X$ as $n \rightarrow \infty$ but either

$$
\lim _{n \rightarrow \infty} \zeta_{M, N}\left(f g x_{n}, g f x_{n}, t\right) \neq 1_{L^{*}}
$$

or the limit does not exist.

Since $f(X) \subset g(X)$, for each $\left\{x_{n}\right\}$, there exists $\left\{y_{n}\right\}$ in $X$ such that $f x_{n}=g y_{n}$. Thus, $f x_{n} \rightarrow z, g x_{n} \rightarrow z$ and $g y_{n} \rightarrow z$ as $n \rightarrow \infty$. By the virtue of this and (56), we obtain $f y_{n} \rightarrow z$. Therefore, we have $f x_{n} \rightarrow z, g x_{n} \rightarrow z, g y_{n} \rightarrow$ $z, f y_{n} \rightarrow z$.

Suppose that $f$ and $g$ are $R$-weakly commuting of type $\left(A_{g}\right)$. Then, weak reciprocal continuity of $f$ and $g$ implies that $f g x_{n} \rightarrow f z$ or $g f x_{n} \rightarrow g z$. Similarly, $f g y_{n} \rightarrow f z$ or $g f y_{n} \rightarrow g z$. Let us first assume that $g f y_{n} \rightarrow g z$. Then, $R$-weak commutativity of type $\left(A_{g}\right)$ of $f$ and $g$ yields

$$
\begin{gathered}
\zeta_{M, N}\left(f f y_{n}, g f y_{n}, t\right) \geq_{L^{*}} \zeta_{M, N}\left(f y_{n}, g y_{n}, \frac{t}{R}\right), \\
\lim _{n \rightarrow \infty} \zeta_{M, N}\left(f f y_{n}, g z, t\right) \geq_{L^{*}} \zeta_{M, N}\left(z, z, \frac{t}{R}\right)=1_{L^{*}}
\end{gathered}
$$

This gives $f f y_{n} \rightarrow g z$. Using (56), we get

$$
\zeta_{M, N}\left(f f y_{n}, f z, k t\right) \geq_{L^{*}} \zeta_{M, N}\left(g f y_{n}, g z, t\right) .
$$

Taking $n \rightarrow \infty$, we have

$$
\zeta_{M, N}(g z, f z, k t) \geq_{L^{*}} \zeta_{M, N}(g z, g z, t)=1_{L^{*}}
$$

This implies that $f z=g z$. Again, by the virtue of $R$-weak commutativity of type $\left(A_{g}\right)$,

$$
\zeta_{M, N}(f f z, g f z, t) \geq_{L^{*}} \zeta_{M, N}\left(f z, g z, \frac{t}{R}\right)=1_{L^{*}}
$$

This yields $f f z=g f z$ and $f f z=f g z=g f z=g g z$. If $f z \neq f f z$, then by using (57), we get

$$
\zeta_{M, N}\left(f z, f^{2} z, t\right)>_{L^{*}} \zeta_{M, N}\left(g z, g^{2} z, t\right)=\zeta_{M, N}\left(f z, f^{2} z, t\right),
$$


which is a contradiction. Hence $f z=f f z=g f z$ and $f z$ is a common fixed point of $f$ and $g$.

Similarly, we can prove that if $f g y_{n} \rightarrow f z$, then, again $f z$ is a common fixed point of $f$ and $g$. Proof is similar if $f$ and $g$ are $R$-weakly commuting of type $\left(A_{f}\right)$ or $R$-weakly commuting of type $(P)$.

We now give an example to illustrate Theorem 27.

Example 28 (see [16]). Let $\left(X, \zeta_{M, N}, \mathscr{T}\right)$ be modified intuitionistic fuzzy metric space as defined in Example 22 and let $\left\{x_{n}\right\}$ be a sequence in $X$ as in Example 22.

Define $f, g: X \rightarrow X$ as follows:

$$
\begin{gathered}
f x=2 \quad \text { if } x=2 \text { or } x>5, \\
f x=4 \quad \text { if } 2<x \leq 5, \\
g 2=2, \quad g x=4 \text { if } 2<x \leq 5, \\
g x=\frac{(x+1)}{3} \quad \text { if } x>5 .
\end{gathered}
$$

Then, $f$ and $g$ satisfy all the conditions of Theorem 27 and have two common fixed points at $x=2$ and $x=4$.

Remark 29. Theorems 23, 25, and 27 generalize the result of Pant et al. [16] for a pair of mappings in modified intuitionistic fuzzy metric space.

\section{Fixed Point and Implicit Relation}

Let $\Theta$ denote the class of those functions $\theta:\left(L^{*}\right)^{5} \rightarrow L^{*}$ such that $\theta$ is continuous and $\theta\left(x, 1_{L^{*}}, 1_{L^{*}}, x, x\right)=x$.

There are examples of $\theta \in \Theta$ :

(1) $\theta_{1}\left(x_{1}, x_{2}, x_{3}, x_{4}, x_{5}\right)=\min \left\{x_{1}, x_{2}, x_{3}, x_{4}, x_{5}\right\}$,

(2) $\theta_{2}\left(x_{1}, x_{2}, x_{3}, x_{4}, x_{5}\right)=x_{1}\left(x_{1}+x_{2}+x_{3}+x_{4}+x_{5}\right) /\left(x_{1}+\right.$ $\left.x_{4}+x_{5}+2 L^{*}\right)$

(3) $\theta_{3}\left(x_{1}, x_{2}, x_{3}, x_{4}, x_{5}\right)=\sqrt[3]{x_{1} x_{4} x_{5}}$.

Now, we prove our results using this implicit relation.

Theorem 30. Let $f$ and $g$ be weakly reciprocally continuous noncompatible self-mappings of a modified intuitionistic fuzzy metric space $\left(X, \zeta_{M, N}, \mathscr{T}\right)$ satisfying the conditions:

$$
\begin{gathered}
f(X) \subset g(X), \\
\int_{0}^{\zeta_{M, N}(f x, f y, t)} \psi(s) d s \geq_{L^{*}} \int_{0}^{\zeta_{M, N}(g x, g y, t)} \psi(s) d s, \\
\int_{0}^{\zeta_{M, N}\left(f x, f^{2} x, t\right)} \psi(s) d s \\
>\int_{0}^{\theta}{\stackrel{\{\zeta}{\left.\zeta_{M, N}(g x, g f x, t), \zeta_{M, N}(f x, g x, t), \zeta_{M, N}\left(g x, f^{2} x, t\right)\right\}}}_{\zeta_{M, N}\left(f^{2} x, g f x, t\right),} \psi(s) d s,
\end{gathered}
$$

whenever $f x \neq f^{2} x$ for all $x, y \in X, t>0$ and for some $\theta \in \Theta$, where $\psi: \mathbb{R}^{+} \rightarrow \mathbb{R}$ is a Lebesgue integrable mapping which is summable nonnegative and such that $\int_{0}^{\varepsilon} \psi(s) d s>0$ for each $\varepsilon>0$.

If $f$ and $g$ are R-weakly commuting of type $\left(A_{g}\right)$ or $R$ weakly commuting of type $\left(A_{f}\right)$ or R-weakly commuting of type $(P)$, then $f$ and $g$ have common fixed point.

Proof. Since $f$ and $g$ are noncompatible maps, there exists a sequence $\left\{x_{n}\right\}$ in $X$ such that $f x_{n} \rightarrow z$ and $g x_{n} \rightarrow z$ for some $z$ in $X$ as $n \rightarrow \infty$ but either $\lim _{n \rightarrow \infty} \zeta_{M, N}\left(f g x_{n}, g f x_{n}, t\right) \neq 1_{L^{*}}$ or the limit does not exist. Since $f(X) \subset g(X)$, for each $\left\{x_{n}\right\}$, there exists $\left\{y_{n}\right\}$ in $X$ such that $f x_{n}=g y_{n}$. Thus, $f x_{n} \rightarrow z, g x_{n} \rightarrow z$ and $g y_{n} \rightarrow z$ as $n \rightarrow \infty$. By the virtue of this and using (66), we obtain

$$
\int_{0}^{\zeta_{M, N}\left(f x_{n}, f y_{n}, t\right)} \psi(s) d s \geq_{L^{*}} \int_{0}^{\zeta_{M, N}\left(g x_{n}, g y_{n}, t\right)} \psi(s) d s
$$

Taking $n \rightarrow \infty$, we have

$$
\int_{0}^{\zeta_{M, N}\left(z, \lim _{n \rightarrow \infty} f y_{n}, t\right)} \psi(s) d s \geq_{L^{*}} \int_{0}^{\zeta_{M, N}(z, z, t)} \psi(s) d s
$$

which implies that $f y_{n} \rightarrow z$ as $n \rightarrow \infty$. Therefore, we have $f x_{n} \rightarrow z, g x_{n} \rightarrow z, g y_{n} \rightarrow z, f y_{n} \rightarrow z$.

Suppose that $f$ and $g$ are $R$-weakly commuting of type $\left(A_{g}\right)$. Then, weak reciprocal continuity of $f$ and $g$ implies that $f g x_{n} \rightarrow f z$ or $g f x_{n} \rightarrow g z$. Similarly, $f g y_{n} \rightarrow f z$ or $g f y_{n} \rightarrow g z$. Let us first assume that $g f y_{n} \rightarrow g z$. Then $R$-weak commutativity of type $\left(A_{g}\right)$ of $f$ and $g$ yields

$$
\begin{gathered}
\zeta_{M, N}\left(f f y_{n}, g f y_{n}, t\right) \geq_{L^{*}} \zeta_{M, N}\left(f y_{n}, g y_{n}, \frac{t}{R}\right), \\
\lim _{n \rightarrow \infty} \zeta_{M, N}\left(f f y_{n}, g z, t\right) \geq_{L^{*}} \zeta_{M, N}\left(z, z, \frac{t}{R}\right)=1_{L^{*}} .
\end{gathered}
$$

This gives $f f y_{n} \rightarrow g z$. Using (66), we get

$$
\int_{0}^{\zeta_{M, N}\left(f f y_{n}, f z, k t\right)} \psi(s) d s \geq_{L^{*}} \int_{0}^{\zeta_{M, N}\left(g f y_{n}, g z, t\right)} \psi(s) d s
$$

Taking $n \rightarrow \infty$, we have

$$
\int_{0}^{\zeta_{M, N}(g z, f z, k t)} \psi(s) d s \geq_{L^{*}} \int_{0}^{\zeta_{M, N}(g z, g z, t)} \psi(s) d s
$$

This implies that $f z=g z$. Again, by the virtue of $R$-weak commutativity of type $\left(A_{g}\right)$,

$$
\zeta_{M, N}(f f z, g f z, t) \geq_{L^{*}} \zeta_{M, N}\left(f z, g z, \frac{t}{R}\right)=1_{L^{*}}
$$


This yields $f f z=g f z$ and $f f z=f g z=g f z=g g z$. If $f z \neq f f z$, then by using (67), we get

$$
\begin{aligned}
& \int_{0}^{\zeta_{M, N}\left(f z, f^{2} z, t\right)} \psi(s) d s \\
& >_{L^{*}} \int_{0}^{\theta} \begin{array}{l}
\left\{\zeta_{M, N}(g z, g f z, t), \zeta_{M, N}(f z, g z, t), \zeta_{M, N}\left(f^{2} z, g f z, t\right),\right. \\
\left.\zeta_{M, N}(f z, g f z, t), \zeta_{M, N}\left(g z, f^{2} z, t\right)\right\}
\end{array}(s) d s \\
& =\int_{0}^{\theta} \begin{array}{l}
\underbrace{}_{M, N} \zeta_{M, N}(f z, f f z, t), \zeta_{M, N}(f z, f z, t), \zeta_{M, N}(f f z, f f z, t), \\
\left.\zeta_{M, N}(f z, f f z, t)\right\}
\end{array}(s) d s \\
& =\int_{0}^{\theta\left\{\zeta_{M, N}\left(f z, f^{2} z, t\right), 1_{L^{*}}, 1_{L^{*}}, \zeta_{M, N}\left(f z, f^{2} z, t\right), \zeta_{M, N}\left(f z, f^{2} z, t\right)\right\}} \psi(s) d s \\
& =\int_{0}^{\zeta_{M, N}\left(f z, f^{2} z, t\right)} \psi(s) d s
\end{aligned}
$$

which is a contradiction. Hence, $f z=f f z=g f z$ and $f z$ is a common fixed point of $f$ and $g$.

Similarly, we can prove if $f g y_{n} \rightarrow f z$, then, again, $f z$ is a common fixed point of $f$ and $g$. Proof is similar if $f$ and $g$ are $R$-weakly commuting of type $\left(A_{f}\right)$ or $(P)$.
If we take $\theta$ as $\theta_{1}, \theta_{2}, \theta_{3}$, then we get the following corollaries.

Corollary 31. Let $f$ and $g$ be weakly reciprocally continuous noncompatible self-mappings of a modified intuitionistic fuzzy metric space $\left(X, \zeta_{M, N}, \mathscr{T}\right)$ satisfying the conditions (65), (66) and

$$
\begin{aligned}
& \int_{0}^{\zeta_{M, N}\left(f x, f^{2} x, t\right)} \psi(s) d s \\
& >_{L^{*}} \int_{0}^{\min \stackrel{\left\{\zeta_{M, N}(g x, g f x, t), \zeta_{M, N}(f x, g x, t), \zeta_{M, N}\left(f^{2} x, g f x, t\right),\right.}{\left.\zeta_{M, N}(f x, g f x, t), \zeta_{M, N}\left(g x, f^{2} x, t\right)\right\}}} \psi(s) d s,
\end{aligned}
$$

whenever $f x \neq f^{2} x$ for all $x, y \in X, t>0$, where $\psi$ : $\mathbb{R}^{+} \rightarrow \mathbb{R}$ is a Lebesgue integrable mapping which is summable nonnegative and such that $\int_{0}^{\varepsilon} \psi(s) d s>0$ for each $\varepsilon>0$.

If $f$ and $g$ are R-weakly commuting of type $\left(A_{g}\right)$ or $R$ weakly commuting of type $\left(A_{f}\right)$ or R-weakly commuting of type $(P)$, then $f$ and $g$ have common fixed point.

Corollary 32. Let $f$ and $g$ be weakly reciprocally continuous noncompatible self-mappings of a modified intuitionistic fuzzy metric space $\left(X, \zeta_{M, N}, \mathscr{T}\right)$ satisfying the conditions (65), (66) and

$$
\begin{aligned}
& \int_{0}^{\zeta_{M, N}\left(f x, f^{2} x, t\right)} \psi(s) d s \\
& >_{L^{*}} \int_{0}^{\zeta_{M, N}(g x, g f x, t)\left(\zeta_{M, N}(g x, g f x, t)+\zeta_{M, N}(f x, g x, t)+\zeta_{M, N}\left(f^{2} x, g f x, t\right)+\zeta_{M, N}(f x, g f x, t)+\zeta_{M, N}\left(g x, f^{2} x, t\right)\right) /\left(\zeta_{M, N}(g x, g f x, t)+\zeta_{M, N}(f x, g f x, t)+\zeta_{M, N}\left(g x, f^{2} x, t\right)+L_{L^{*}}\right)} \psi(s) d s,
\end{aligned}
$$

whenever $f x \neq f^{2} x$ for all $x, y \in X, t>0$, where $\psi$ : $\mathbb{R}^{+} \rightarrow \mathbb{R}$ is a Lebesgue integrable mapping which is summable nonnegative and such that $\int_{0}^{\varepsilon} \psi(s) d s>0$ for each $\varepsilon>0$.

If $f$ and $g$ are $R$-weakly commuting of type $\left(A_{g}\right)$ or $R$ weakly commuting of type $\left(A_{f}\right)$ or R-weakly commuting of type $(P)$, then $f$ and $g$ have common fixed point.

Corollary 33. Let $f$ and $g$ be weakly reciprocally continuous noncompatible self-mappings of a modified intuitionistic fuzzy metric space $\left(X, \zeta_{M, N}, \mathscr{T}\right)$ satisfying the conditions (65), (66), and

$$
\begin{aligned}
& \int_{0}^{\zeta_{M, N}\left(f x, f^{2} x, t\right)} \psi(s) d s \\
& \quad>\int_{0}^{\sqrt[3]{\zeta_{M, N}(g x, g f x, t) \cdot \zeta_{M, N}(f x, g f x, t) \cdot \zeta_{M, N}\left(g x, f^{2} x, t\right)}} \psi(s) d s,
\end{aligned}
$$

whenever $f x \neq f^{2} x$ for all $x, y \in X, t>0$, where $\psi$ : $\mathbb{R}^{+} \rightarrow \mathbb{R}$ is a Lebesgue integrable mapping which is summable nonnegative and such that $\int_{0}^{\varepsilon} \psi(s) d s>0$ for each $\varepsilon>0$.
If $f$ and $g$ are $R$-weakly commuting of type $\left(A_{g}\right)$ or $R$ weakly commuting of type $\left(A_{f}\right)$ or $R$-weakly commuting of type $(P)$, then $f$ and $g$ have common fixed point.

Let $\Delta$ denote the class of those functions $\delta:\left(L^{*}\right)^{4} \rightarrow L^{*}$ such that $\delta$ is continuous and $\delta\left(x, 1_{L^{*}}, x, 1_{L^{*}}\right)=x$.

There are examples of $\delta \in \Delta$ :

(1) $\delta_{1}\left(x_{1}, x_{2}, x_{3}, x_{4}\right)=\min \left\{x_{1}, x_{2}, x_{3}, x_{4}\right\}$,

(2) $\delta_{2}\left(x_{1}, x_{2}, x_{3}, x_{4}\right)=\sqrt{x_{1} x_{3}}$.

Now, we prove our main results.

Theorem 34. Let $f$ and $g$ be weakly reciprocally continuous noncompatible self-mappings of a modified intuitionistic fuzzy metric space $\left(X, \zeta_{M, N}, \mathscr{T}\right)$ satisfying the conditions (65), (66), and

$$
\begin{aligned}
& \int_{0}^{\zeta_{M, N}\left(f x, f^{2} x, t\right)} \psi(s) d s \\
& >_{L^{*}} \int_{0}^{\delta}{ }_{\left.\zeta_{M, N}\left(f^{2} x, g f f x, t\right)\right)}^{\left(\zeta_{M x}(f x, g x, t), \zeta_{M, N}(f x, g f x, t),\right.}, \psi(s) d s,
\end{aligned}
$$


whenever $f x \neq f^{2} x$ for all $x, y \in X, t>0$ and for some $\delta \in \Delta$ where $\psi: \mathbb{R}^{+} \rightarrow \mathbb{R}$ is a Lebesgue integrable mapping which is summable nonnegative and such that $\int_{0}^{\varepsilon} \psi(s) d s>0$ for each $\varepsilon>0$.

If $f$ and $g$ are $R$-weakly commuting of type $\left(A_{g}\right)$ or $R$ weakly commuting of type $\left(A_{f}\right)$ or R-weakly commuting of type $(P)$, then $f$ and $g$ have common fixed point.

Proof. Since $f$ and $g$ are noncompatible maps, there exists a sequence $\left\{x_{n}\right\}$ in $X$ such that $f x_{n} \rightarrow z$ and $g x_{n} \rightarrow$ $z$ for some $z$ in $X$ as $n \rightarrow \infty$ but either $\lim _{n \rightarrow \infty}$ $\zeta_{M, N}\left(f g x_{n}, g f x_{n}, t\right) \neq 1_{L^{*}}$ or the limit does not exist. Since $f(X) \subset g(X)$, for each $\left\{x_{n}\right\}$, there exists $\left\{y_{n}\right\}$ in $X$ such that $f x_{n}=g y_{n}$. Thus $f x_{n} \rightarrow z, g x_{n} \rightarrow z$ and $g y_{n} \rightarrow z$ as $n \rightarrow \infty$. By the virtue of this and using (78), we obtain

$$
\int_{0}^{\zeta_{M, N}\left(f x_{n}, f y_{n}, t\right)} \psi(s) d s \geq_{L^{*}} \int_{0}^{\zeta_{M, N}\left(g x_{n}, g y_{n}, t\right)} \psi(s) d s
$$

Taking $n \rightarrow \infty$, we have

$$
\int_{0}^{\zeta_{M, N}\left(z, \lim _{n \rightarrow \infty} f y_{n}, t\right)} \psi(s) d s \geq_{L^{*}} \int_{0}^{\zeta_{M, N}(z, z, t)} \psi(s) d s
$$

which implies that, $f y_{n} \rightarrow z$ as $n \rightarrow \infty$. Therefore, we have $f x_{n} \rightarrow z, g x_{n} \rightarrow z, g y_{n} \rightarrow z, f y_{n} \rightarrow z$.

Suppose that $f$ and $g$ are $R$-weakly commuting of type $\left(A_{g}\right)$. Then, weak reciprocal continuity of $f$ and $g$ implies that $f g x_{n} \rightarrow f z$ or $g f x_{n} \rightarrow g z$. Similarly, $f g y_{n} \rightarrow f z$ or $g f y_{n} \rightarrow g z$. Let us first assume that $g f y_{n} \rightarrow g z$. Then $R$-weak commutativity of type $\left(A_{g}\right)$ of $f$ and $g$ yields

$$
\begin{gathered}
\zeta_{M, N}\left(f f y_{n}, g f y_{n}, t\right) \geq_{L^{*}} \zeta_{M, N}\left(f y_{n}, g y_{n}, \frac{t}{R}\right), \\
\lim _{n \rightarrow \infty} \zeta_{M, N}\left(f f y_{n}, g z, t\right) \geq_{L^{*}} \zeta_{M, N}\left(z, z, \frac{t}{R}\right)=1_{L^{*}}
\end{gathered}
$$

This gives $f f y_{n} \rightarrow g z$. Using (78), we get

$$
\int_{0}^{\zeta_{M, N}\left(f f y_{n}, f z, k t\right)} \psi(s) d s \geq_{L^{*}} \int_{0}^{\zeta_{M, N}\left(g f y_{n}, g z, t\right)} \psi(s) d s
$$

Taking $n \rightarrow \infty$, we have

$$
\int_{0}^{\zeta_{M, N}(g z, f z, k t)} \psi(s) d s \geq_{L^{*}} \int_{0}^{\zeta_{M, N}(g z, g z, t)} \psi(s) d s
$$

This implies that $f z=g z$. Again, by the virtue of $R$ weak commutativity of type $\left(A_{g}\right), \zeta_{M, N}(f f z, g f z, t) \geq_{L^{*}} \zeta_{M, N}$ $(f z, g z, t / R)=1_{L^{*}}$. This yields $f f z=g f z$ and $f f z=f g z=$ $g f z=g g z$. If $f z \neq f f z$, then by using (78), we get

$$
\begin{aligned}
& \int_{0}^{\zeta_{M, N}\left(f z, f^{2} z, t\right)} \psi(s) d s \\
& >_{L^{*}} \int_{0}^{\delta} \zeta_{M, N}^{\left(\zeta_{M, N}(g z, g f z, t), \zeta_{M, N}(f z, g z, t), \zeta_{M, N}(f z, g f z, t),\right.}, \quad \psi(s) d s \\
& =\int_{0}^{\delta} \zeta_{M, N}^{\left(\zeta_{M, N}\left(f z, f^{2} z, t\right), \zeta_{M, N}(f z, f z, t), \zeta_{M, N}\left(f z, f^{2} z, t\right),\right.} \psi(s) d s \\
& =\int_{0}^{\delta\left(\zeta_{M, N}\left(f z, f^{2} z, t\right), 1_{L^{*}}, \zeta_{M, N}\left(f z, f^{2} z, t\right), 1_{L^{*}}\right)} \psi(s) d s \\
& =\int_{0}^{\zeta_{M, N}\left(f z, f^{2} z, t\right)} \psi(s) d s,
\end{aligned}
$$

which is a contradiction. Hence, $f z=f f z=g f z$ and $f z$ is a common fixed point of $f$ and $g$.

Similarly, we can prove that if $f g y_{n} \rightarrow f z$, then, again, $f z$ is a common fixed point of $f$ and $g$. Proof is similar if $f$ and $g$ are $R$-weakly commuting of type $\left(A_{f}\right)$ or $(P)$.

If we take $\delta$ as $\delta_{1}, \delta_{2}$, then we get the following corollaries.

Corollary 35. Let $f$ and $g$ be weakly reciprocally continuous noncompatible self-mappings of a modified intuitionistic fuzzy metric space $\left(X, \zeta_{M, N}, \mathscr{T}\right)$ satisfying the conditions (65), (66), and

$$
\begin{aligned}
& \int_{0}^{\zeta_{M, N}\left(f x, f^{2} x, t\right)} \psi(s) d s
\end{aligned}
$$

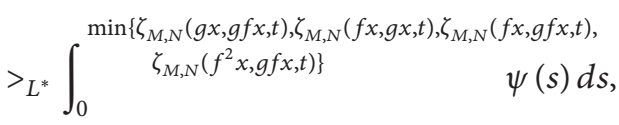

whenever $f x \neq f^{2} x$ for all $x, y \in X, t>0$, where $\psi$ : $\mathbb{R}^{+} \rightarrow \mathbb{R}$ is a Lebesgue integrable mapping which is summable nonnegative and such that $\int_{0}^{\varepsilon} \psi(s) d s>0$ for each $\varepsilon>0$.

If $f$ and $g$ are $R$-weakly commuting of type $\left(A_{g}\right)$ or $R$ weakly commuting of type $\left(A_{f}\right)$ or $R$-weakly commuting of type $(P)$, then $f$ and $g$ have common fixed point.

Corollary 36. Let $f$ and $g$ be weakly reciprocally continuous noncompatible self-mappings of a modified intuitionistic fuzzy metric space $\left(X, \zeta_{M, N}, \mathscr{T}\right)$ satisfying the conditions (65), (66) and

$$
\int_{0}^{\zeta_{M, N}\left(f x, f^{2} x, t\right)} \psi(s) d s>_{L^{*}} \int_{0}^{\sqrt{\zeta_{M, N}(g x, g f x, t) \cdot \zeta_{M, N}(f x, g f x, t)}} \psi(s) d s,
$$

whenever $f x \neq f^{2} x$ for all $x, y \in X, t>0$ and for some $\delta \in \Delta$, where $\psi: \mathbb{R}^{+} \rightarrow \mathbb{R}$ is a Lebesgue integrable mapping which is summable nonnegative and such that $\int_{0}^{\varepsilon} \psi(s) d s>0$ for each $\varepsilon>0$. 
If $f$ and $g$ are $R$-weakly commuting of type $\left(A_{g}\right)$ or $R$ weakly commuting of type $\left(A_{f}\right)$ or $R$-weakly commuting of type $(P)$, then $f$ and $g$ have common fixed point.

\section{References}

[1] R. Saadati, S. Sedghi, and N. Shobe, "Modified intuitionistic fuzzy metric spaces and some fixed point theorems," Chaos, Solitons \& Fractals, vol. 38, no. 1, pp. 36-47, 2008.

[2] S. Jain, S. Jain, and L. Bahadur Jain, "Compatibility of type (P) in modified intuitionistic fuzzy metric space," Journal of Nonlinear Science and its Applications, vol. 3, no. 2, pp. 96-109, 2010.

[3] S. Sedghi, N. Shobe, and A. Aliouche, "Common fixed point theorems in intuitionistic fuzzy metric spaces through conditions of integral type," Applied Mathematics \& Information Sciences, vol. 2, no. 1, pp. 61-82, 2008.

[4] H. Adibi, Y. J. Cho, D. O’Regan, and R. Saadati, "Common fixed point theorems in L-fuzzy metric spaces," Applied Mathematics and Computation, vol. 182, no. 1, pp. 820-828, 2006.

[5] S. Manro, S. Kumar, S. Kumar, and S. S. Bhatia, "Common fixed point theorem in intuitionistic fuzzy metric spaces using common (E.A) property and implicit relation," Journal of Advanced Studies in Topology, vol. 3, no. 3, pp. 60-68, 2012.

[6] R. Saadati, S. Sedghi, and H. Zhou, "A common fixed point theorem for $\psi$-weakly commuting maps in $L$-metric spaces," Iranian Journal of Fuzzy Systems, vol. 5, no. 1, p. 47-53, 103, 2008.

[7] R. Saadati, S. M. Vaezpour, and Y. J. Cho, "Quicksort algorithm: application of a fixed point theorem in intuitionistic fuzzy quasi-metric spaces at a domain of words," Journal of Computational and Applied Mathematics, vol. 228, no. 1, pp. 219225, 2009.

[8] G. Jungck, "Compatible mappings and common fixed points," International Journal of Mathematics and Mathematical Sciences, vol. 9, no. 4, pp. 771-779, 1986.

[9] R. P. Pant, "A common fixed point theorem under a new condition," Indian Journal of Pure and Applied Mathematics, vol. 30, no. 2, pp. 147-152, 1999.

[10] G. Deschrijver and E. E. Kerre, "On the relationship between some extensions of fuzzy set theory," Fuzzy Sets and Systems, vol. 133, no. 2, pp. 227-235, 2003.

[11] G. Deschrijver, C. Cornelis, and E. E. Kerre, "On the representation of intuitionistic fuzzy $t$-norm and $t$-conorm," IEEE Transactions on Fuzzy Systems, vol. 12, no. 1, pp. 45-61, 2004.

[12] R. Saadati and J. H. Park, "On the intuitionistic fuzzy topological spaces," Chaos, Solitons \& Fractals, vol. 27, no. 2, pp. 331-344, 2006.

[13] M. Tanveer, M. Imdad, D. Gopal, and D. Kumar, "Common fixed point theorem in modified Intuitionistic fuzzy metric spaces with common property (E.A.)," Fixed Point Theory and Applications, vol. 36, 2012.

[14] R. P. Pant, "Common fixed points of noncommuting mappings," Journal of Mathematical Analysis and Applications, vol. 188, no. 2, pp. 436-440, 1994.

[15] H. K. Pathak, Y. J. Cho, and S. M. Kang, "Remarks on $R$-weakly commuting mappings and common fixed point theorems," Bulletin of the Korean Mathematical Society, vol. 34, no. 2, pp. 247-257, 1997.

[16] R. P. Pant, R. K. Bisht, and D. Arora, "Weak reciprocal continuity and fixed point theorems," Annali dell'Universitá di Ferrara, vol. 57, no. 1, pp. 181-190, 2011. 


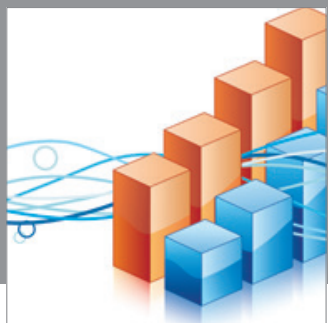

Advances in

Operations Research

mansans

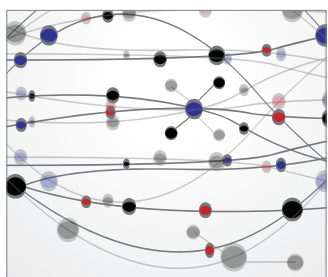

The Scientific World Journal
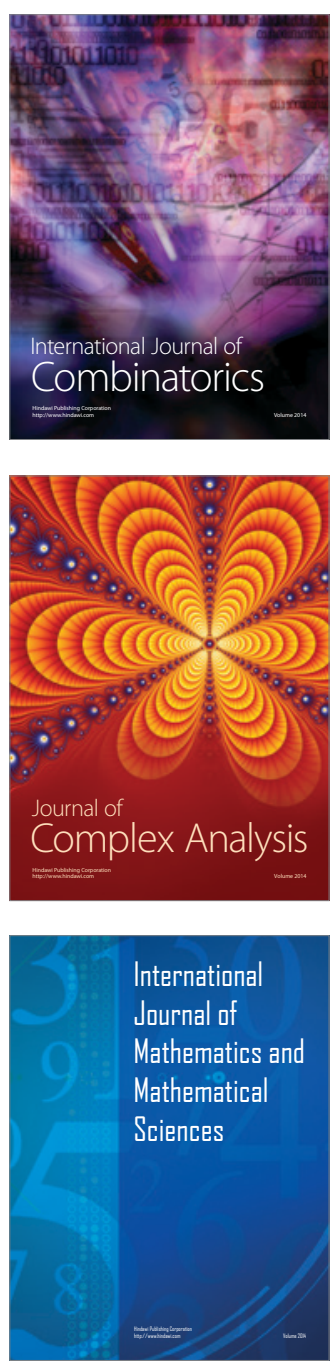
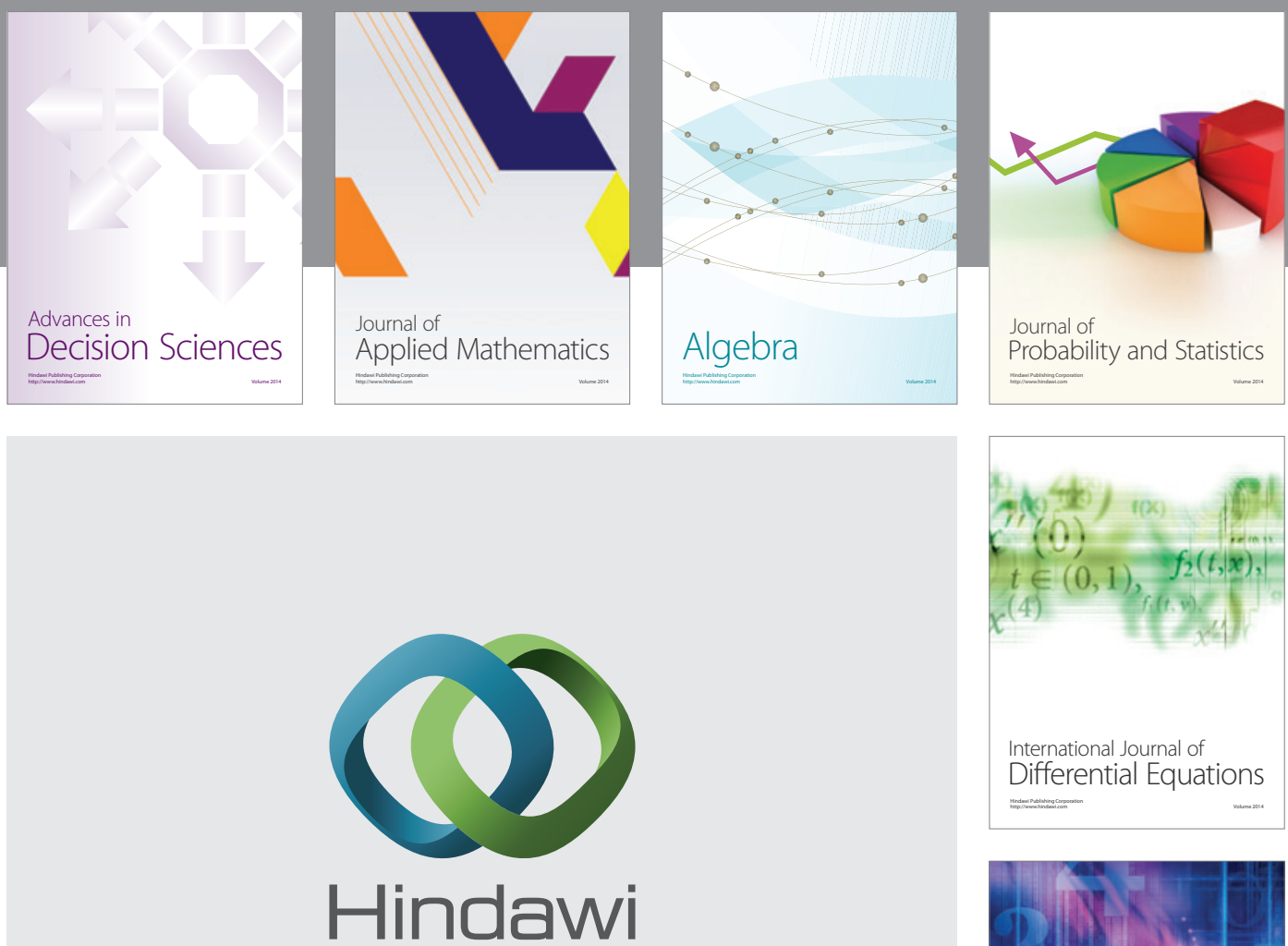

Submit your manuscripts at http://www.hindawi.com
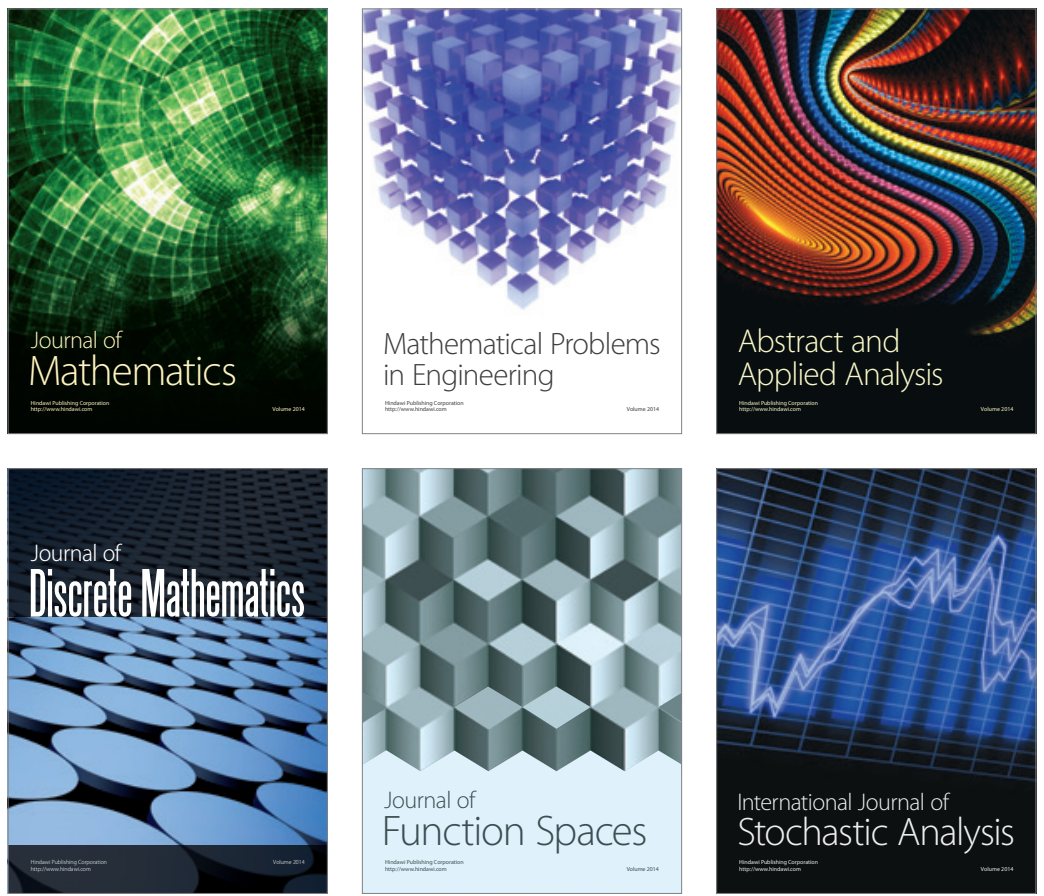

Journal of

Function Spaces

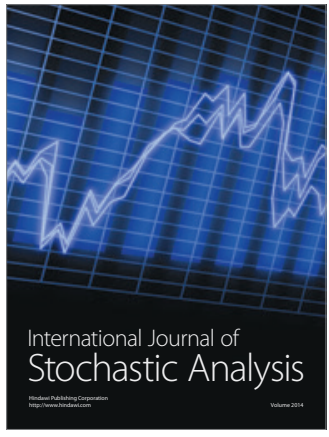

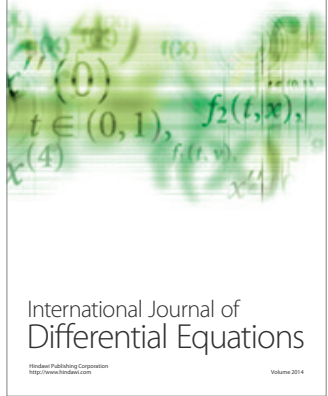
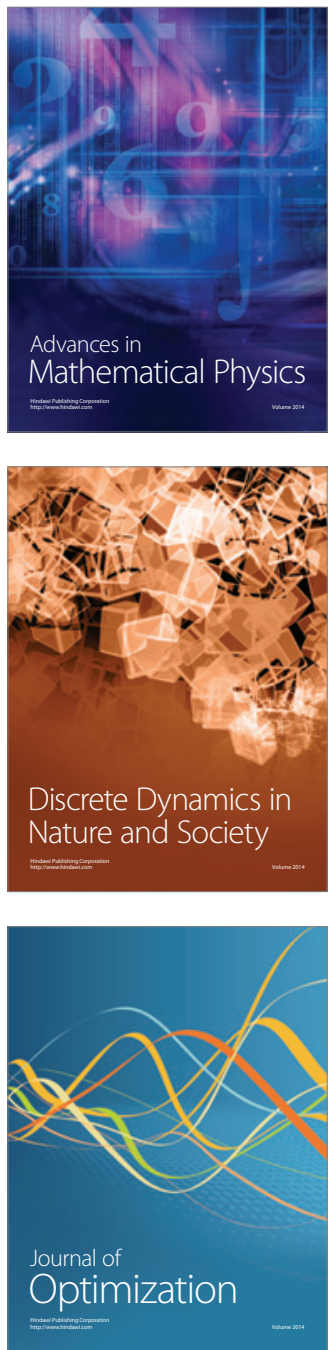